\title{
Private States, Quantum Data Hiding and the Swapping of Perfect Secrecy
}

\author{
Matthias Christandl* and Roberto Ferrara ${ }^{\dagger}$ \\ QMATH, Department of Mathematical Sciences, University of Copenhagen, \\ Universitetsparken 5, 2100 Copenhagen Ø, Denmark
}

(Dated: February 21, 2018)

\begin{abstract}
An important contribution to the understanding of quantum key distribution has been the discovery of entangled states from which secret bits, but no maximally entangled states, can be extracted [Horodecki et al., Phys. Rev. Lett. 94, 200501 (2005)]. The construction of those states was based on an intuition that the quantum mechanical phenomena of data hiding and privacy might be related. In this Letter we firmly connect these two phenomena and highlight three aspects of this result. First, we simplify the definition of the secret key rate. Second, we give a formula for the one-way distillable entanglement of certain private states. Third, we consider the problem of extending the distance of quantum key distribution with help of intermediate stations, a setting called the quantum key repeater. We show that for protocols that first distill private states, it is essentially optimal to use the standard quantum repeater protocol based on entanglement distillation and entanglement swapping.
\end{abstract}

\section{INTRODUCTION}

Entanglement distillation [1] is the process of producing high-fidelity maximally entangled states from copies of a noisy entangled state $\rho$, using only Local Operations and Classical Communication (LOCC), between two parties Alice and Bob. The maximally entangled states can then be used for teleportation, Bell inequality violation, etc. The rate at which they can be distilled from $\rho$ is called the distillable entanglement, $E_{D}(\rho)$. Because maximally entangled states are pure, they are in product with the environment and, therefore, measuring them leads to perfectly correlated and perfectly secure pairs of bits, the perfect secret bits. It turns out that there exist mixed states, the private states, that also lead to perfectly secure bits just by measurement [2]. While the distillable key, $K_{D}(\rho)$, is defined as the rate at which perfect secret bits can be distilled by local operations and public communication, it was shown that it also equals the rate at which private states can be distilled by LOCC. Proving this equivalence allowed the authors to show that distillable entanglement and distillable key can be very different [2]. There even exists a low-dimensional experimental realization of this separation with photonic states [3].

In light of this, it is natural to ask how much the separation extends to general network scenarios, and in particular whether it persists if we insert a repeater station between the two parties. In [4] the first examples have been produced of states that, while having high distillable key, do not allow for distillation of significant amounts of the key across the repeater station. This may be an indication that the separation between the distillable key and distillable entanglement does not survive in all general network scenarios.

\footnotetext{
* christandl@math.ku.dk

† roberto@math.ku.dk
}

Here we provide a new perspective on key distillation, and thus quantum key distribution, by relating private states to quantum data hiding $[5,6]$. This provides a tool for the study of long-distance quantum key distribution involving intermediate repeater stations, where for the first time we are able to show a close connection with entanglement distillation. In this framework [4], noisy entanglement is distributed between the end points and the repeater station and arbitrary noiseless LOCC protocols are allowed. If this setting is used to distill maximally entangled states at the end points then this is an idealized version of the well-known quantum repeater and if it is used to distill private states it is called a quantum key repeater. We provide an upper bound on the quantum key repeater rate with one-way classical communication; as such, the bound holds also for noisy protocols that can only lower the rate and thus, if anything, leave room for improvement. Our results go beyond the use of the partial transpose and thus apply to states that are not positive under partial transposition (NPT states) as well as states that are invariant under partial transposition (PPT invariant states), which are out of reach for [4].

The Letter is organized as follows. First, we simplify the class of private states, introducing what we call Bell private states. We show that these states are, for all entanglement-related purposes, equivalent to private states. Second, the simplified structure of Bell private states allows us to confirm the intuition that the separation between the distillable key and distillable entanglement is due to quantum data hiding. More precisely, we show that the states with a separation are those made of a maximally entangled state subject to phase flip error, where the error information is conserved in data-hiding states. Such hidden information of the error preserves the key, but prevents Alice and Bob from correcting the maximally entangled state and distill entanglement. Third, as an application to the quantum key repeater with one-way classical communication from the repeater station, we show that a large class of states and protocols cannot be used to distill the key across a repeater station better than 
by performing entanglement distillation and swapping.

\section{PRIVATE STATES}

Consider two parties Alice and Bob sharing a maximally entangled state $\Phi$ of two qubit systems $A_{k} B_{k}$, the key systems. Measuring $\Phi$ in the computational basis will produce a perfect secret bit with respect to any adversary; the postmeasurement state of such a measurement is called a key attacked state, this will play an important role in our results and will be denoted by a hat $\left(^{\wedge}\right)$ :

$$
\begin{aligned}
& \Phi:=\frac{1}{2}(|00\rangle+|11\rangle)(\langle 00|+\langle 11|) \\
& \hat{\Phi}=\frac{1}{2}(|00\rangle\langle 00|+| 11\rangle\langle 11|) .
\end{aligned}
$$

The support of $\hat{\Phi}$ is known as the maximally correlated subspace. Now let Alice and Bob share additional systems $A_{s} B_{s}$, the shield systems. A private state $\gamma$ is a state on $A_{k} B_{k} A_{s} B_{s}$ that generalizes the maximally entangled state, in the sense that measuring $A_{k} B_{k}$ produces a perfect secret bit with respect to any adversary. $\gamma$ is a private state if and only if it has the form [2]:

$$
\gamma:=T(\Phi \otimes \sigma) T^{\dagger} \quad \hat{\gamma}=T(\hat{\Phi} \otimes \sigma) T^{\dagger} .
$$

for some state $\sigma$ on $A_{s} B_{s}$ and controlled unitary $T$ called twisting; with no shield systems the only private states are maximally entangled states. However, the first example of a private states with low distillable entanglement was constructed as follows [2]:

$$
\gamma=p_{0} \cdot \mathcal{Z}_{B_{k}}^{0}(\Phi) \otimes \sigma_{0}+p_{1} \cdot \mathcal{Z}_{B_{k}}^{1}(\Phi) \otimes \sigma_{1}
$$

where $\sigma_{j}$ are the extremal Werner states [7] and $\mathcal{Z}^{j}(\varrho):=$ $Z^{j} \varrho Z^{-j}$ is the $j$ th phase flip map, namely the map that conjugates by the $j$ th power of the Pauli $Z$. The intuition behind the example is the following: orthogonal datahiding states $\sigma_{j}[5,6]$, like the Werner states [8], should hinder the ability to correct the phase flip locally and, thus, they should suppress the distillable entanglement, nevertheless, because the states are orthogonal, the perfect secret bit is still protected from the environment.

Private states like the ones in Equation (3) are only a special case (see [9] for different examples); we call them Bell private states. We now show how to convert all private states into Bell private states reversibly using only LOCC. We need two generalizations.

We generalize the maximally entangled state to any key systems of equal finite dimension $\left|A_{k}\right|=\left|B_{k}\right|$. We define the Bell states $\phi_{j}=\left|\phi_{j}\right\rangle\left\langle\phi_{j}\right|=\mathcal{Z}_{B_{k}}^{j}(\Phi)$ for $j=$ $0, \ldots,\left|B_{k}\right|-1$. Notice that $\left\{\left|\phi_{j}\right\rangle\right\}$ form a basis for the maximally correlated subspace, which brings us to the next generalization. We consider any state supported only on the maximally correlated subspace of $A_{k} B_{k}$, we call such states key correlated. They have no bit-flip error

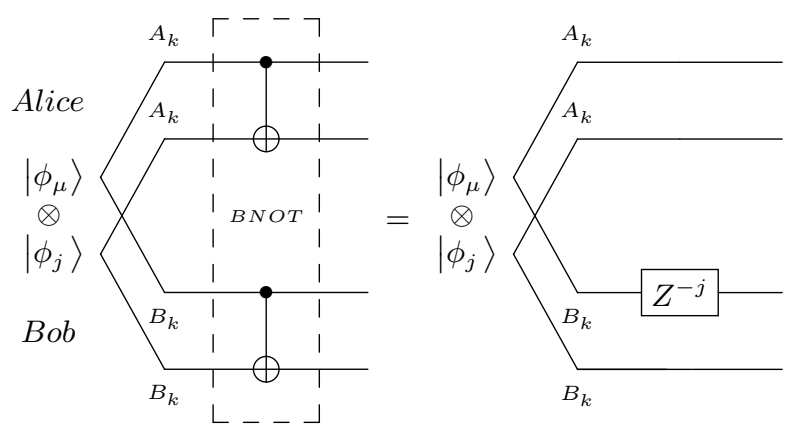

FIG. 1. Quantum circuit for the Bilateral CNOT acting on Bell states, the core of the map $\mathcal{E}$ of Lemma 1 .

and we can write them as:

$$
\rho=\sum_{\mu \nu}\left|\phi_{\mu}\right\rangle\left\langle\phi_{\nu}\right| \otimes P_{\mu \nu},
$$

where $P_{\mu \nu}$ are matrices on $A_{s} B_{s}$.

To define the reversible LOCC map, consider two copies of systems $A_{k} B_{k}$. Let $V=C N O T_{A_{k} A_{k}} \otimes C N O T_{B_{k} B_{k}}$ be the local unitary illustrated in Figure 1, namely the generalization of the qubit BNOT [1]. It holds that:

$$
V\left(\left|\phi_{j}\right\rangle_{A_{k} B_{k}} \otimes\left|\phi_{\mu}\right\rangle_{A_{k} B_{k}}\right)=\left|\phi_{j}\right\rangle_{A_{k} B_{k}} \otimes Z_{B_{k}}^{-j}\left|\phi_{\mu}\right\rangle_{A_{k} B_{k}} .
$$

Lemma 1. Define $\mathcal{E}: A_{k} B_{k} \rightarrow A_{k} B_{k} A_{k} B_{k}$ as

$$
\mathcal{E}\left(\varrho_{A_{k} B_{k}}\right):=V^{\dagger}\left(\hat{\Phi}_{A_{k} B_{k}} \otimes \varrho_{A_{k} B_{k}}\right) V .
$$

Then for any key correlated state $\rho$ (on $A_{k} B_{k} A_{s} B_{s}$ ):

$$
\mathcal{E}(\rho) \equiv\left(\mathcal{E}_{A_{k} B_{k}} \otimes \operatorname{id}_{A_{s} B_{s}}\right)(\rho)=\frac{1}{\left|B_{k}\right|} \sum_{j} \phi_{j} \otimes \mathcal{Z}_{B_{k}}^{j}(\rho) .
$$

Because $\hat{\Phi}$ is separable and $V$ is local, $\mathcal{E}$ is one-way LOCC (classical communication only from Alice to Bob or vice versa). $\mathcal{E}$ is reversible by inverting $V$ and tracing out the target, which requires only local operations. Notice that the output key systems are still $A_{k} B_{k}$ but the output shield systems are now $A_{k} A_{s} B_{k} B_{s}$.

Proof. Using (4), (5) and $\hat{\Phi}=\frac{1}{\left|B_{k}\right|} \sum_{j} \phi_{j}$ we find

$$
\begin{aligned}
(\mathcal{E} \otimes \mathrm{id})(\rho) & =\sum_{\mu \nu} V^{\dagger}\left(\hat{\Phi} \otimes\left|\phi_{\mu}\right\rangle \phi_{\nu} \mid\right) V \otimes P_{\mu \nu} \\
& =\frac{1}{\left|B_{k}\right|} \sum_{\mu \nu j} V^{\dagger}\left(\phi_{j} \otimes\left|\phi_{\mu}\right\rangle \phi_{\nu} \mid\right) V \otimes P_{\mu \nu} \\
& =\frac{1}{\left|B_{k}\right|} \sum_{\mu \nu j} \phi_{j} \otimes \mathcal{Z}_{B_{k}}^{j}\left(\left|\phi_{\mu}\right\rangle\left\langle\phi_{\nu}\right|\right) \otimes P_{\mu \nu}
\end{aligned}
$$

Bell private states now come as a special case. A Bell private state is any private state of the form:

$$
\gamma_{B e l l}=\sum_{j} p_{j} \cdot \phi_{j} \otimes \sigma_{j}
$$


where $\sigma_{j}$ are arbitrary orthogonal states of $A_{s} B_{s}$ and $p_{j}$ are arbitrary probabilities. Notice that $\mathcal{Z}_{B_{k}}^{j}(\rho)$ are orthogonal, and thus $\mathcal{E}(\rho)$ is a Bell private state, only when $\rho$ is a private state. Because $\mathcal{E}$ is reversible, any property of entanglement monotones (entanglement measures like the distillable entanglement and distillable key) for Bell private states also holds for private states and vice versa. For example, we can always convert the output of a key distillation protocol into an approximate Bell private state, thus simplifying the distillable key to the rate at which Bell private states can be distilled.

\section{ENTANGLEMENT DISTILLATION AND QUANTUM DATA HIDING}

We now show that Bell private states with low distillable entanglement are states that hide the phase of the maximally entangled states from local detection. Specifically, we give a lower bound on the one-way distillable entanglement $E_{D}(\rho)$, where the communication is one way from Alice to Bob. This lower bound is the rate achieved by the best protocol that starts with a measurement on Alice's shield.

First for simplicity, let Alice and Bob share a key correlated state of the form

$$
\rho=\frac{1}{\left|B_{k}\right|} \sum_{j} \phi_{j} \otimes \sigma_{j}
$$

We now let Alice perform a measurement on her shield and send the outcome to Bob. Then Alice and Bob use the hashing protocol $[1,10]$ and we find:

$$
E_{D}(\rho) \geq \sup _{\mathcal{M} \in L O_{A}} \frac{1}{\left|B_{k}\right|} \sum_{j} D\left(\mathcal{M}\left(\sigma_{j}\right) \| \mathcal{M}(\sigma)\right)
$$

where $\sigma=\frac{1}{\left|B_{k}\right|} \sum_{j} \sigma_{j}, D(\varrho \| \varsigma)=\operatorname{tr}[\varrho \log \varrho-\rho \log \varsigma]$ is the relative entropy, and $\mathcal{M}$ is a local measurement at Alice $\left(\mathcal{M} \equiv \mathcal{M}_{A} \otimes \operatorname{id}_{B}\right)$. See also the Appendix Lemma 18 for the details. The relative entropy quantifies the distinguishability between states; the relative entropy of the measurement outcomes [11] quantifies how much of this distinguishability is left when Alice and Bob can only act locally. In the particular case of private states, the $\sigma_{j}$ states of Equation (7) are orthogonal; thus, they are perfectly distinguishable and $j$ can be recovered with a global measurement. However, Equation (8) implies that if the distillable entanglement is low, then the local distinguishability of $\sigma_{j}$ is low and $j$ cannot be determined accurately locally: the $\sigma_{j}$ are data hiding $[5,6]$.

For general key correlated states $\rho$ we can use Equation (8) after using Lemma 1, this gives

$$
E_{\vec{D}}(\rho) \geq \sup _{\mathcal{M} \in L O_{A}} \frac{1}{\left|B_{k}\right|} \sum_{j} D\left(\mathcal{M}\left(\mathcal{Z}_{B_{k}}^{j}(\rho)\right) \| \mathcal{M}(\hat{\rho})\right) .
$$

Namely we see that because of the reversible map, we can think of the private state itself as a data-hiding state, where $j$ is encoded using the local phase flip.

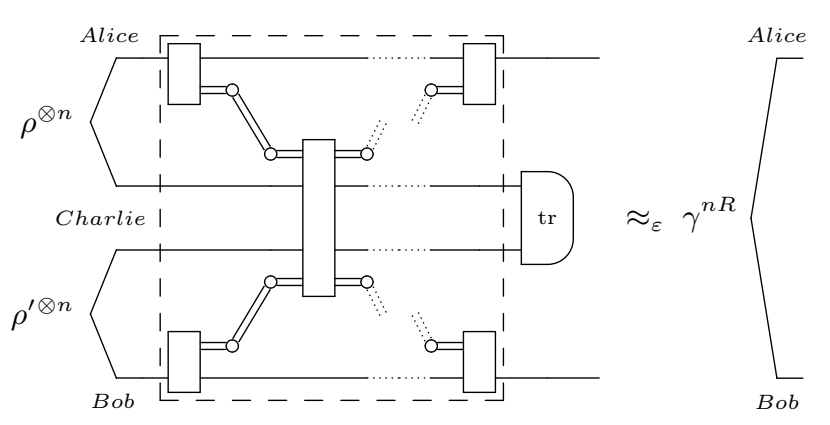

FIG. 2. Quantum circuit for the key repeater protocols in a single node repeater. The dashed box is a tripartite LOCC protocol. The double lines are the classical communication.

We can exploit the measurement being local to simplify our bounds. More precisely, we find that for all local measurements at Alice

$$
D\left(\mathcal{M}\left(\mathcal{Z}_{B_{k}}^{j}(\rho)\right) \| \mathcal{M}(\hat{\rho})\right)=D(\mathcal{M}(\rho) \| \mathcal{M}(\hat{\rho})) .
$$

Namely, the optimal measurement is independent of the phase flip, which allows us to remove the phase flip in the formula. This is an important feature because it suddenly allows us to regularize $[12,13]$ our lower bound. If $\hat{\rho}$ is separable, then we can combine the regularized lower bound with a known upper bound from [14], and obtain equality with the distillable entanglement as stated in this theorem (see the Appendix Theorem 21 for the details).

Theorem 2. For any key correlated state $\rho$, it holds

$$
\begin{aligned}
& E_{D}^{\vec{D}}(\rho) \geq D_{A}(\rho \| \hat{\rho}):=\sup _{\mathcal{M} \in L O_{A}} D(\mathcal{M}(\rho) \| \mathcal{M}(\hat{\rho})) \\
& E_{D}(\rho) \geq D_{A}^{\infty}(\rho \| \hat{\rho}):=\lim _{n \rightarrow \infty} \frac{1}{n} D_{A}\left(\rho^{\otimes n} \| \hat{\rho}^{\otimes n}\right)
\end{aligned}
$$

If $\hat{\rho}$ is also separable then:

$$
E_{D}(\rho)=D_{A}^{\infty}(\rho \| \hat{\rho}) .
$$

\section{QUANTUM KEY REPEATERS}

We now apply our findings to long-distance quantum communication, where noise prevents Alice and Bob from sharing entanglement and thus secrecy, and where an intermediate repeater station, Charlie, is necessary to mediate the entanglement.

More precisely, let Alice and Charlie $(A$ and $C)$ share $\rho$ and Charlie and Bob $\left(C^{\prime}\right.$ and $\left.B\right)$ share $\rho^{\prime}$. While the goal of a quantum repeater is to distill maximally entangled states between Alice and Bob [15], the goal of a quantum key repeater is to distill perfect secret bits or, equivalently, private states [4], see Figure 2. The best rate for this task is called the quantum key repeater rate, $R_{D}\left(\rho, \rho^{\prime}\right)$. Realistic repeaters have multiple stations; however, we reduce to a single station by grouping them into one, 
which can only increase the rate. The reduction to a single station thus provides upper bounds without loss of generality.

$\rho$ and $\rho^{\prime}$ are usually generated by sharing maximally entangled states through noisy channels (Choi-Jamiołkowski states). While clever channel codes may reach higher rates $[16,17]$, note that the free classical side information allows us in most realistic channels to implement the codes via teleportation from the Choi-Jamiołkowski state (e.g. depolarizing channel) [1]. Thus our upper bounds also apply to such codes and channels (see also [18]).

The optimal noise-free protocol for the quantum repeater performs entanglement distillation between Alice and Charlie, and between Charlie and Bob, followed by entanglement swapping. This results in the rate $\min \left\{E_{D}(\rho), E_{D}\left(\rho^{\prime}\right)\right\}$, but in the quantum key repeater setting the situation is less clear. In alternative to the mentioned protocol, Alice and Charlie can distill private states, and use the maximally entangled states distilled by Charlie and Bob to teleport Charlie's part of the private states. If $E_{D}\left(\rho^{\prime}\right)$ is larger than the private states size at Charlie's, then the rate of this "trivial" protocol equals $K_{D}(\rho)$ and thus it will be positive even when $\rho$ has zero distillable entanglement $[2,9]$. In short, while for quantum repeaters the active area of research studies the effect of noisy operations, for quantum key repeaters there are open questions even with perfect operations.

We will consider the one-way key repeater rate variation, also introduced in [4]. In this variation, Alice and Bob's communication with the repeater station Charlie is only one way: Charlie can send messages to Alice and Bob but not vice versa. Alice and Bob can still communicate normally with each other. We denote this rate with $R_{D}^{C C^{\prime} \rightarrow A: B}$, or simply $R_{D}$. In [4] the question was posed whether there exist non-trivial protocols beyond distillation and swapping, but only negative examples were found. Here we show that for a large class of states and protocols, the one-way distillable entanglement is an upper bound on the one-way key repeater rate, and thus distillation and swapping are essentially optimal and far from the trivial upper bounds $K_{D}(\rho)$ and $K_{D}\left(\rho^{\prime}\right)$.

We need a general upper bound which follows from [4, Theorem 4]:

$$
R_{D}^{C C^{\prime} \rightarrow A: B}\left(\rho_{A C}, \rho_{C^{\prime} B}^{\prime}\right) \leq D_{C C^{\prime}}^{\infty}\left(\rho_{A C} \otimes \rho_{C^{\prime} B}^{\prime} \| \sigma\right)
$$

for any state $\sigma$ separable in the $A C C^{\prime}: B$ or $A: C C^{\prime} B$ cut. So far, this bound could only be estimated via a relaxation that only works for states that are PPT (Positive under Partial Transposition). Choosing $\sigma=$ $\hat{\rho} \otimes \hat{\rho}^{\prime}$ and applying Theorem 2 to Equation (9) now shows the following corollary, independently of the partial transpose.

Corollary 3. For any key correlated states $\rho$ and $\rho^{\prime}$ with at least one separable key attacked state, it holds:

$$
R_{D}^{C C^{\prime} \rightarrow A: B}\left(\rho_{A C}, \rho_{C^{\prime} B}^{\prime}\right) \leq E_{D}^{C \rightarrow A}\left(\rho_{A C} \otimes \rho_{C A}^{\prime}\right) .
$$

Since all private states are NPT (Non-positive under Partial Transposition) [19], this gives the first examples of NPT states with a high distillable key but low one-way key repeater rate.

Example. Consider the following Bell private state (see [2] and Equation (3)):

$$
\gamma=\frac{1}{2}\left(1+\frac{1}{d}\right) \phi_{0} \otimes \sigma_{0}+\frac{1}{2}\left(1-\frac{1}{d}\right) \phi_{1} \otimes \sigma_{1}
$$

where $\sigma_{0}$ and $\sigma_{1}$ are, respectively, the symmetric and anti-symmetric states in $\mathbb{C}^{d} \otimes \mathbb{C}^{d}$ (the extreme Werner states [7] which are known to be data-hiding states [8]). Since distillable entanglement is upper bounded by the $\log$-negativity $E_{N}[20]$, we have the following upper bound which vanishes for large $d$ :

$$
R_{D}(\gamma, \gamma) \leq 2 E_{D}^{\rightarrow}(\gamma) \leq 2 E_{N}(\gamma)=2 \log \left(1+\frac{1}{d}\right)
$$

This state was implemented experimentally for $d=2[3]$. The key was distilled at a rate $K \approx 0.69$, enough to break the bound at $E_{N}(\gamma)=\log \frac{3}{2} \approx 0.58$. However, because of the factor of 2 in Equation (10), an implementation with $d=4$ at the same key rate is required for the same proof of concept. Still, scaling up the implementation should be experimentally feasible, since in $d=4$ the gate used (swap) is tensor product of qubit gates. In the Appendix Example 41 we show how to apply Corollary 3 to some PPT invariant states.

\section{CONCLUSIONS}

Corollary 3 bounds the key repeater rate of a restricted class of states, but it also generalizes to all states if we restrict the protocols to first distill private states with separable key attacked state between the nodes and then try to repeat. In the Appendix Definition 28 we define a new key repeater rate $R_{D}^{\rangle}\left(\rho, \rho^{\prime}\right)$ from these protocols and prove that for all states, this rate is upper bounded by $E_{D}\left(\rho \otimes \rho^{\prime}\right)$. The restricted protocols still include one-way entanglement distillation and swapping; thus, the new key repeater rate is still lower bounded by the minimum of the one-way distillable entanglements. While being restrictive, we would like to stress that the communication between Alice and Bob is two way, and also that if the two-way step is limited to bipartite distillation between the nodes, we can always apply the result to the outcomes of the distillation. In particular even if the two-way recurrence protocol is used to distill between the nodes, as in the case of heralded entanglement generation and purification, we can apply the bound on $\left\langle R_{D}^{\succ}\right.$ to the outputs of the recurrence protocol. The bound also applies to key repeater schemes based on quantum error correction. The link with outgoing communication from the station is trivially covered. For the link with incoming communication, the bound on $R_{D}^{\rangle}$applies to the output of the code (as mentioned above), since usually the code is decoded or corrected at the station rendering it a bipartite distillation protocol. As such, we can 
apply our bound in some way to most repeater schemes (see also [17] and references therein for an overview) and where it applies, any attempt to improve the rate of key distillation above that of entanglement distillation will not work. For example, attempting to use the noisy processing protocol[21] would yield no advantage. We are not aware that there exist any protocol that contains a truly two-way tripartite step.

Finally, we note that, because optimal one-way protocols exist when close to the target states, the optimal two-way protocols are composed of a two-way "lift-off", protocol followed by a one-way "conclusion" protocol [22].

We leave as an open problem whether Corollary 3 generalizes to all states and protocols, including two-way communication. Such a result would show that all entangled states with zero distillable entanglement, including those with distillable key, have zero key repeater rate. Another open problem, called the $\mathrm{PPT}^{2}$ conjecture [23], asks whether swapping PPT states in all dimensions always yields separable states. If the conjecture is true, then it would imply that all PPT states have zero key repeater rate. In that, the results here presented support the conjecture. Since our results are asymptotic in nature, they give a complementary view on the $\mathrm{PPT}^{2}$ conjecture to that of the study of swapping specific states in specific dimensions.

The connection made between key distillation, entanglement distillation and quantum data hiding raises the possibility of finding a rate at which data hiding states can be distilled, $H_{D}$ (which we refrain from defining formally). Namely, in performing entanglement distillation on private states, it may be possible to retain the undistillable correlations into data hiding states with zero distillable entanglement so that they could be used as a resource, such that

$$
K_{D}(\rho)=H_{D}(\rho)+E_{D}(\rho)
$$

\section{ACKNOWLEDGMENTS}

We thank Alexander Müller-Hermes, Cécilia Lancien, and Māris Ozols for helpful discussions. We acknowledge financial support from the European Research Council (ERC Grant Agreement No. 337603), the Danish Council for Independent Research (Sapere Aude), and VILLUM FONDEN via the QMATH Centre of Excellence (Grant No. 10059).
[1] C. Bennett, D. DiVincenzo, J. Smolin, W. Wootters, Mixed-state entanglement and quantum error correction. Phys. Rev. A 54, 3824 (1996), arXiv: quant-ph/9604024.

[2] K. Horodecki, M. Horodecki, P. Horodecki, J. Oppenheim, Secure key from bound entanglement. Phys. Rev. Lett. 94, 160502 (2005), arXiv: quant-ph/0309110.

[3] K. Dobek, M. Karpiński, R. Demkowicz-Dobrzański, K. Banaszek, P. Horodecki, Experimental Extraction of Secure Correlations from a Noisy Private State. Phys. Rev. Lett. 106, 030501 (2011), arXiv: 1010.4575.

[4] S. Bäuml, M. Christandl, K. Horodecki, A. Winter, Limitations on Quantum Key Repeaters. Nat. Commun. 6, 6908 (2014), arXiv: 1402.5927.

[5] B. Terhal, D. DiVincenzo, D. Leung, Hiding bits in Bell states. Phys. Rev. Lett. 86, 5807 (2001), arXiv: quant$\mathrm{ph} / 0011042$.

[6] D. DiVincenzo, B. Terhal, D. Leung, Quantum data hiding. IEEE Trans. Inf. Theory 48, No. 3, 580 (2002). arXiv: quant-ph/0103098.

[7] R. Werner, Quantum states with Einstein-PodolskyRosen correlations admitting a hidden-variable model. Phys. Rev. A 40, 4277 (1989).

[8] T. Eggeling, R. Werner Hiding classical data in multipartite quantum states. Phys. Rev. Lett. 89, 097905 (2002), arXiv: quant-ph/0203004.

[9] K. Horodecki, Ł. Pankowski, M. Horodecki, P. Horodecki, Low dimensional bound entanglement with one-way distillable cryptographic key. IEEE Trans. Inf. Theory 54, 2621 (2008), arXiv: quant-ph/0506203.

[10] I. Devetak, A. Winter, Distillation of secret key and entanglement from quantum states. Proc. R. Soc. Lond. A 461, 207 (2005), arXiv: quant-ph/0306078.

[11] M. Piani, Relative Entropy of Entanglement and Re- stricted Measurements. Phys. Rev. Lett. 103, 160504 (2009), arXiv: 0904.2705.

[12] M. Fekete, Über die Verteilung der Wurzeln bei gewissen algebraischen Gleichungen mit ganzzahligen Koeffizienten. Math. Z. 17, (1) 228 (1923).

[13] P. Hayden, M. Horodecki, B. Terhal, The asymptotic entanglement cost of preparing a quantum state. J. Phys. A 34, (35) 6891 (2001), arXiv: quant-ph/0008134.

[14] K. Li, A. Winter, Relative entropy and squashed entanglement. Commun. Math. Phys. 326, (1) 63 (2014), arXiv: 1210.3181.

[15] H. Briegel, W. Dür, J. Cirac, P. Zoller, Quantum Repeaters: The Role of Imperfect Local Operations in Quantum Communication. Phys. Rev. Lett. 81, 5932 (1998), arXiv: quant-ph/9803056.

[16] E. Knill, R. Laflamme, Concatenated Quantum Codes. arXiv: quant-ph/9608012.

[17] S. Muralidharan, L. Li, J. Kim, N. Lütkenhaus, M. Lukin, L. Jiang, Optimal architectures for long distance quantum communication. Sci. Rep. 6, 20463 (2016).

[18] M. Christandl, A. Müller-Hermes, Relative Entropy Bounds on Quantum, Private and Repeater Capacities. Commun. Math. Phys. 353, 821 (2017), arXiv: 1604.03448 .

[19] K. Horodecki, M. Horodecki, P. Horodecki, J. Oppenheim, General paradigms for distilling classical key from quantum states. IEEE Trans. Inf. Theory 55, 1898 (2009), arXiv: quant-ph/0506189.

[20] G. Vidal, R. Werner, Computable measure of entanglement. Phys. Rev. A 65, 032314 (2002), arXiv: quant$\mathrm{ph} / 0102117$.

[21] R. Renner, N. Gisin, B. Kraus, Information-theoretic security proof for quantum-key-distribution protocols. Phys. 
Rev. A 72, 012332 (2005), arXiv: quant-ph/0502064.

[22] D. Kretschmann, R. Werner, Tema con variazioni: quantum channel capacity. New J. Phys. 6, 26 (2004) arXiv: quant-ph/0311037.

[23] M. Christandl, PPT square conjecture (problem G), in Banff International Research Station workshop: Operator structures in quantum information theory, https://www.birs.ca/workshops/2012/12w5084/ report12w5084.pdf.

[24] D. Petz, Sufficient subalgebras and the relative entropy of states of a von Neumann algebra. Commun. Math. Phys. 105, (1) 123 (1986).

[25] V. Vedral, M. Plenio, M. Rippin, P. Knight, Quantifying entanglement. Phys. Rev. Lett. 78, 122275 (1997), arXiv: quant-ph/9702027.

[26] M. Horodecki, P. Horodecki, R. Horodecki, Mixed-state entanglement and distillation: is there a "bound" entanglement in nature?. Phys. Rev. Lett. 80, 5239 (1998), arXiv: quant-ph/9801069.

[27] M. Donald, M. Horodecki, Continuity of Relative Entropy of Entanglement. Phys. Lett. A 264, 1999 (257), arXiv: quant-ph/9910002.

[28] K. Audenaert, B. De Moor, K. Vollbrecht, R. Werner, Asymptotic Relative Entropy of Entanglement for Orthogonally Invariant States. Phys. Rev. A 66, 032310 (2002), arXiv: quant-ph/0204143.

[29] P. Badziag, M. Horodecki, A. Sen, U. Sen, Locally accessible information: How much can the parties gain by cooperating?. Phys. Rev. Lett. 91, 117901 (2003), arXiv: quant-ph/0304040.

[30] R. Alicki, M. Fannes, Continuity of quantum conditional information. J. Phys. A 37, L55 (2004), arXiv: quant$\mathrm{ph} / 0312081$.

[31] T. Hiroshima, M. Hayashi, Finding a maximally correlated state - Simultaneous Schmidt decomposition of bipartite pure states. Phys. Rev. A 70, 030302 (2004), arXiv: quant-ph/0405107.

[32] K. Horodecki, M. Horodecki, P. Horodecki, J. Oppenheim, Locking entanglement measures with a single qubit. Phys. Rev. Lett. 94, 200501 (2005), arXiv: quant-ph/0404096.

[33] A. Winter, Tight Uniform Continuity Bounds for Quantum Entropies: Conditional Entropy, Relative Entropy Distance and Energy Constraints. Commun. Math. Phys. 347, 291 (2016), arXiv: 1507.07775.

[34] M. Berta, O. Fawzi, M. Tomamichel, On Variational Expressions for Quantum Relative Entropies, in 2016 IEEE International Symposium on Information Theory (ISIT), (IEEE, Barcelona, 2016) pp. 2844-2848. arXiv: 1512.02615 .

\section{APPENDICES}

In these appendices we present the background concepts used in this article, we show how to further apply our findings to more complex repeater scenarios and we provide more examples.

We begin with a review of the generalized Bell states and of their properties, especially with respect to the bilateral CNOT which is fundamental to the reversible map. Then we analyse Bell private states further and present some minor properties.
After this, we move onto entanglement measures. First we review the various entanglement measures based on the relative entropy and its restriction to quantum measurements; this heavily relies on the work made in [11]. We then explain all the distillation rates mentioned in this paper and give their explicit definition, this includes the various forms of distillable entanglement, distillable key and key repeater rate. We also discuss in details some known upper bounds on these distillation rates that were used in the main text.

With these concepts in place we present further applications of our results: the one-way key swapper (a novel repeater rate) and the single-copy repeater rate. Finally, we give further examples of states with vanishing one-way key repeater rate, which include NPT states, PPT states and PPT invariant states.

Last but not least, the reader can find an exhaustive list of notations in Table 6 and Table 7.

\section{Appendix A: Generalized Bell states}

Consider a two qubit system $\left(\mathbb{C}^{2} \otimes \mathbb{C}^{2}\right)$ with the maximally entangled state:

$$
|\Phi\rangle:=\frac{1}{\sqrt{2}}(|00\rangle+|11\rangle) .
$$

We can write the Bell states as bit flips and phase flips of the maximally entangled state where the bit flip and phase flip unitaries are

$$
X:=\left(\begin{array}{cc}
0 & 1 \\
1 & 0
\end{array}\right) \quad Z:=\left(\begin{array}{cc}
1 & 0 \\
0 & -1
\end{array}\right),
$$

and they generate the Bell states when acting on a single qubit of a maximally entangled state. In general, acting on one qubit or the other yields different bases. We will choose to act always on the first qubit and so we define

$$
\left|\phi_{i j}\right\rangle:=\left(X^{i} Z^{j} \otimes \mathbb{1}\right)|\Phi\rangle \quad i, j=0,1
$$

which gives the Bell states:

$$
\begin{aligned}
\left|\phi_{00}\right\rangle & =\frac{1}{\sqrt{2}}(|00\rangle+|11\rangle)=|\Phi\rangle \\
\left|\phi_{01}\right\rangle & =\frac{1}{\sqrt{2}}(|00\rangle-|11\rangle) \\
\left|\phi_{10}\right\rangle & =\frac{1}{\sqrt{2}}(|10\rangle+|01\rangle) \\
\left|\phi_{11}\right\rangle & =\frac{1}{\sqrt{2}}(|10\rangle-|01\rangle) .
\end{aligned}
$$

The generalized Bell states are defined in a similar way. We consider now a two qudit system $\left(\mathbb{C}^{d} \otimes \mathbb{C}^{d}\right)$ whose maximally entangled state is now:

$$
|\Phi\rangle=\frac{1}{d} \sum_{i \in \mathbb{Z}_{d}}|i i\rangle,
$$


where $\mathbb{Z}_{d}=\mathbb{Z} / d \mathbb{Z} \equiv\{0, \ldots, d-1\}$ is the cyclic additive group of order $d$ on the integers, namely the integers with addition modulo $d: i+j=i+j \bmod d$. The unitary generalization of the bit and phase flip are:

$$
X:=\sum_{j \in \mathbb{Z}_{d}}|j+1\rangle\left\langle j\left|, \quad Z:=\sum_{j \in \mathbb{Z}_{d}} \omega^{j}\right| j\right\rangle\langle j|,
$$

where $\omega=e^{i \frac{2 \pi}{d}}$ is the $d^{\prime}$ th root of unity. Just as before, the Bell states are now defined using powers of $X$ and $Z$.

\section{Definition 4 (Generalized Bell states).}

$$
\left|\phi_{i j}\right\rangle:=\left(X^{i} Z^{j} \otimes \mathbb{1}\right)|\Phi\rangle
$$

for $i, j=\mathbb{Z}_{d}$.

Finally, we further define the following notation for the density matrix of these states:

$$
\Phi:=|\Phi\rangle\left\langle\Phi\left|, \quad \phi_{i j}=\right| \phi_{i j}\right\rangle\left\langle\phi_{i j}\right| .
$$

As mentioned in the main text, we denote with the hat $\left(^{\wedge}\right)$ the operation that measures the key systems in the computational basis. For the case of the maximally entangled state this measurement yields the maximally correlated state:

$$
\hat{\Phi}=\frac{1}{d} \sum_{i \in \mathbb{Z}_{d}}|i i\rangle\langle i i|
$$

The maximally correlated state is a uniform mixture of orthogonal pure states, we thus define the maximally correlated subspace as the support of the maximally correlated state. We call $\mathbb{1}_{\hat{\Phi}}:=d \hat{\Phi}$ the projector onto this subspace. Measuring any of $\phi_{0 j}$ (the Bell states with phase flips only) in the computational basis yields the same maximally correlated state

$$
\hat{\phi}_{0 j}=\hat{\Phi} \quad \forall j
$$

and $\mathbb{1}_{\hat{\Phi}}$ can be written as a mixture of Bell states in the following way:

$$
\begin{aligned}
\mathbb{1}_{\hat{\Phi}} & =\sum_{i \in \mathbb{Z}_{d}}|i i\rangle\left\langle i i\left|=\sum_{i, j \in \mathbb{Z}_{d}} \delta_{i j}\right| i i\right\rangle\langle j j| \\
& =\sum_{i, j, k \in \mathbb{Z}_{d}} \frac{1}{d} \omega^{k(i-j)}|i i\rangle\left\langle j j\left|=\sum_{k \in \mathbb{Z}_{d}}\right| \phi_{0 k}\right\rangle\left\langle\phi_{0 k}\right| . \\
& =\sum_{k \in \mathbb{Z}_{d}} \phi_{0 k} .
\end{aligned}
$$

For the remainder of the article, we will omit $\mathbb{Z}_{d}$ and, unless otherwise stated, all indexes will be summed over the cyclic group, where the order is given by the dimension of the corresponding Hilbert space.

The bilateral CNOT is a gate of four systems obtained by applying two CNOT gates, two of the systems will be the controls and the others will be the targets. In [1] it
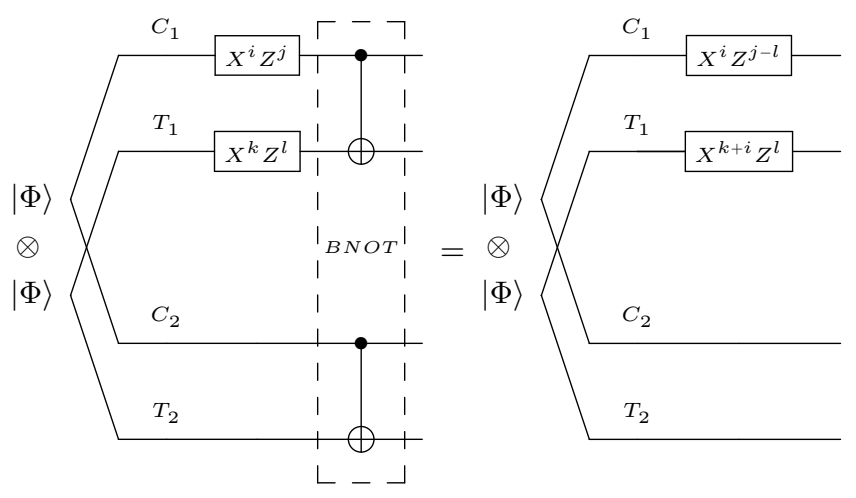

FIG. 3. Effect of the BNOT on Bell states, see Lemma 5.
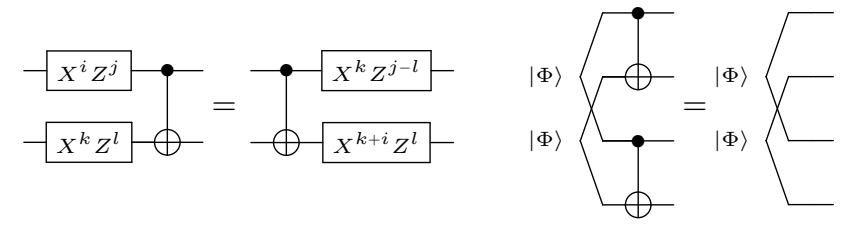

FIG. 4. Effect of commuting CNOT with arbitrary bit/phase flips.

FIG. 5. Bell states with no bit/phase flips are invariant under BNOT.

was called Bilateral XOR (BXOR) and it was defined only for qubit systems. Here we use the generalized CNOT on $\mathbb{C}^{d} \otimes \mathbb{C}^{d}:$

$$
C N O T:=\sum_{a b}|a, a+b\rangle\langle a b| .
$$

The straightforward generalization of the BXOR gate, the bilateral CNOT, is then:

$$
B N O T_{C T}:=C_{N O T} T_{C_{1} T_{1}} \otimes C N O T_{C_{2} T_{2}}
$$

where systems $C=C_{1} C_{2}$ are the control qudits, systems $T=T_{1} T_{2}$ are the target qudits and all qudits have the same size, i.e. $\left|C_{1}\right|=\left|C_{2}\right|=\left|T_{1}\right|=\left|T_{2}\right|=d$. Like in [1], our interest in the gate lies in its effect on Bell states. Notice how the BNOT is a local operation as long as the system is partitioned as $C_{1} T_{1}: C_{2} T_{2}$.

Lemma 5. For all $i, j, k, l \in \mathbb{Z}_{d}$ :

$$
B N O T_{C T} \cdot\left|\phi_{i j}\right\rangle_{C} \otimes\left|\phi_{k l}\right\rangle_{T}=\left|\phi_{i, j-l}\right\rangle_{C} \otimes\left|\phi_{k+i, l}\right\rangle_{T}
$$

Proof.

$$
\begin{aligned}
& \text { BNOT } \cdot\left|\phi_{i j}\right\rangle \otimes\left|\phi_{k l}\right\rangle \\
& =B N O T \cdot \frac{1}{d} \sum_{a b} \omega^{j a}|a+i, a\rangle \otimes \omega^{l b}|b+k, b\rangle \\
& =\frac{1}{d} \sum_{a b} \omega^{j a}|a+i, a\rangle \otimes \omega^{l b}|b+k+a+i, b+a\rangle .
\end{aligned}
$$


Now we make a change of variable $\tilde{b}=b+a$ :

$$
\begin{aligned}
& =\frac{1}{d} \sum_{a \tilde{b}} \omega^{j a}|a+i, a\rangle \otimes \omega^{l(\tilde{b}-a)}|\tilde{b}+k+i, \tilde{b}\rangle \\
& =\frac{1}{d} \sum_{a \tilde{b}} \omega^{(j-l) a}|a+i, a\rangle \otimes \omega^{l \tilde{b}}|\tilde{b}+k+i, \tilde{b}\rangle \\
& =\left|\phi_{i, j-l}\right\rangle \otimes\left|\phi_{k+i, l}\right\rangle .
\end{aligned}
$$

An alternative and maybe more intuitive way to prove Lemma 5 , is to notice that $\Phi \otimes \Phi$ is invariant under the action of the BNOT, see Figure 5, and that CNOT is a Clifford gate with a simple update rule, see Figure 4. Namely, it holds that

$$
B N O T_{C T} \cdot\left(|\Phi\rangle_{C} \otimes|\Phi\rangle_{T}\right)=|\Phi\rangle_{C} \otimes|\Phi\rangle_{T}
$$

and

$$
C N O T \cdot X^{i} Z^{j} \otimes X^{k} Z^{l}=X^{i} Z^{j-l} \otimes X^{i+k} Z^{l} \cdot C N O T^{\dagger} .
$$

Applying Equation (A1) and Equation (A2) to Definition 4 proves Lemma 5 as displayed in Figure 3. From Lemma 5, it follows in particular that

$$
B N O T^{\dagger} \cdot \phi_{00} \otimes \phi_{0 j} \cdot B N O T=\phi_{0 j} \otimes \phi_{0 j}
$$

which is what we use in Lemma 1 (see main text).

\section{Appendix B: Bell private states}

The maximally entangled states produce perfect secret bits with respect to the environment when measured in the computational basis. The private states are those states that generalize this property, namely they are all those states that produce perfect secret bits with respect to the environment when measured in the computational basis. Let us recall that a state is a private state if and only if it can be written as[2]:

$$
\gamma^{m}:=T(\Phi \otimes \sigma) T^{\dagger}
$$

where $m=\log \left|A_{k}\right|=\log \left|B_{k}\right|, T$ is a controlled unitary $T=\sum_{i j}|i j\rangle\langle i j| \otimes U_{i}$, and $\sigma$ is an arbitrary state. It is implicit that $\Phi$ is a state of $A_{k} B_{k}$ and $\sigma$ is a state of $A_{s} B_{s}$. Let us also recall the definition of Bell private states:

$$
\gamma_{\text {Bell }}^{m}:=\sum_{j \in \mathbb{Z}_{2} m} p_{j} \phi_{0 j} \otimes \sigma_{j}
$$

where $\sigma_{j}$ are arbitrary orthogonal states. Again $\phi_{i j}$ are states of $A_{k} B_{k}$ and $\sigma_{j}$ are states of $A_{s} B_{s}$.

All Bell private states are private states. This can be proved either by checking that the measurement in $A_{k} B_{k}$ gives perfectly secure bits or by showing that they admit an expression as private states, here we show the latter.
Lemma 6. A state $\gamma_{B e l l}^{m}$ is a Bell private state if and only if it can be written as $\gamma_{\text {Bell }}^{m}=T(\Phi \otimes \sigma) T^{\dagger}$ using

$$
T=\sum_{i j}|i j\rangle\langle i j| \otimes U_{\sigma}^{i} \quad \sigma=\sum_{j} p_{j} \sigma_{j}
$$

where $U_{\sigma}^{i}$ is the $i$-th power of

$$
U_{\sigma}=\sum_{j} \omega^{j} P_{\sigma_{j}}+P_{\sigma_{\perp}}
$$

with $P_{\sigma_{j}}$ the projectors onto the supports of $\sigma_{j}$ and $P_{\sigma_{\perp}}=$ $\mathbb{1}-\sum_{j} P_{\sigma_{j}}$ the remaining orthogonal projector.

$P_{\sigma_{\perp}}$ plays no active role, it is only needed to complete $T$, so that $\sigma$ is not required to have full support.

Proof. The following sequence of equalities proves that a state is of the form $\sum p_{j} \phi_{0 j} \otimes \sigma_{j}$, thus a Bell private state, if and only if it is of the form $T(\Phi \otimes \sigma) T^{\dagger}$ with $\sigma$ and $T$ as above.

$$
\begin{aligned}
\gamma^{m} & =\sum_{k} p_{k} \phi_{0 k} \otimes \sigma_{k} \\
& =\sum_{i j k} p_{k} \frac{1}{2^{m}} \omega^{i k}|i i\rangle\langle j j| \omega^{-j k} \otimes P_{\sigma_{k}} \sigma_{k} P_{\sigma_{k}} \\
& =\sum_{i j k} \frac{1}{2^{m}}|i i\rangle\langle j j| \otimes\left(\omega^{i k} P_{\sigma_{k}}\right) \cdot\left(p_{k} \sigma_{k}\right) \cdot\left(\omega^{-j k} P_{\sigma_{k}}\right) \\
& =\sum_{i j k \alpha \beta} \frac{1}{2^{m}}|i i\rangle\langle j j| \otimes\left(\omega^{i \alpha} P_{\sigma_{\alpha}}\right) \cdot\left(p_{k} \sigma_{k}\right) \cdot\left(\omega^{-j \beta} P_{\sigma_{\beta}}\right) \\
& =\sum_{i j} \frac{1}{2^{m}}|i i\rangle\langle j j| \otimes \\
& =\sum_{i j} \frac{1}{2^{m}}|i i\rangle\langle j j| \otimes U_{\sigma}^{i} \sigma U_{\sigma}^{-j} \\
& =\sum_{i j}\left(|i\rangle\langle i| \otimes U_{\sigma}^{i}\right) \cdot\left(\frac{1}{2^{m}}|i i\rangle\langle j j| \otimes \sigma\right) \cdot\left(|j\rangle\langle j| \otimes U_{\sigma}^{j \dagger}\right) \\
& =T\left(\Phi^{m} \otimes \sigma\right) P^{\dagger}
\end{aligned}
$$

where we used the orthogonality of the $\sigma_{k}$ in the identity

$$
\sum_{k \alpha} P_{\sigma_{\alpha}} \sigma_{k}=\sum_{k} P_{\sigma_{k}} \sigma_{k} .
$$

For $\left|A_{k}\right|=\left|B_{k}\right|=2$, any private state admits a block form [19], namely it can be written as:

$$
\gamma^{1}=\frac{1}{2}\left(\begin{array}{cccc}
\sqrt{Y^{\dagger} Y} & 0 & 0 & Y^{\dagger} \\
0 & 0 & 0 & 0 \\
0 & 0 & 0 & 0 \\
Y & 0 & 0 & \sqrt{Y Y^{\dagger}}
\end{array}\right)
$$

where $Y$ is any opportune matrix of unit trace norm $\left(\|Y\|_{1}=1\right)$. This can be easily seen by recalling that any matrix $Y$ admits a singular value decomposition and noticing that the decomposition can be used to extract 
$\sigma$ and the unitaries $U_{0}$ and $U_{1}$ in $T$. However, this does not work in higher dimension $\left(m=\log \left|A_{k}\right|>1\right)$ because then additional unitaries are needed to specify $T$.

This is not true for Bell private states, indeed, a Bell private state only needs to specify a single unitary $U_{\sigma}$. This allows one to write a block form for all private states by exploiting the fact that $U_{\sigma}$ and $\sigma$ commute.

Corollary 7. $\gamma_{\text {Bell }}^{m}$ is a Bell private state iff

$$
\gamma_{\text {Bell }}^{m}=\frac{1}{2^{m}} \sum_{i j}|i i\rangle\langle j j|\otimes| Y|\left(\frac{Y}{|Y|}\right)^{i-j}
$$

for some normal $Y\left(Y^{\dagger} Y=Y Y^{\dagger}\right)$ such that $\|Y\|_{1}=1$ and $Y^{2^{m}} \geq 0$.

Proof. Set $Y=\sigma U_{\sigma}$. Then $|Y|=\sigma, \frac{Y}{|Y|}=U_{\sigma}$ and

$$
U_{\sigma}^{i} \sigma U_{\sigma}^{-j}=\sigma U_{\sigma}^{i-j}=|Y|\left(\frac{Y}{|Y|}\right)^{i-j} .
$$

In Corollary 7, $Y^{-1}$ is intended as pseudo inversion of matrices so that $Y$ need not to be full rank. Note how in the corollary $m=1$ the corollary implies $Y=Y^{\dagger}$.

We now consider some simple entropic properties of private states. For this purpose let us introduce the following state

$$
\stackrel{\gamma}{\gamma}=\gamma_{A_{k} B_{k}} \otimes \gamma_{A_{s} B_{s}}
$$

namely the tensor product of the key and shield marginals of a private state. For Bell private states this has a form similar to the key attacked state:

$$
\stackrel{\circ}{\text { Bell }}=\sum_{j} p_{j} \phi_{0 j} \otimes \sigma
$$

which indeed gives $\dot{\gamma}_{\text {Bell }}=\hat{\gamma}_{\text {Bell }}$ for uniform probability distributions $\left\{p_{k}\right\}$.

We can summarize the difference between $\dot{\gamma}$ and the key attacked state $\hat{\gamma}$ using quantum relative entropies $(D(\rho \| \sigma)=\operatorname{tr}[\rho \log \rho-\rho \log \sigma])$ as follows:

$$
\begin{aligned}
& D\left(\gamma_{\text {Bell }}^{m} \| \hat{\gamma}_{\text {Bell }}^{m}\right)=D(\Phi \| \hat{\Phi})=m \\
& D\left(\gamma_{\text {Bell }} \| \stackrel{\gamma}{\text { Bell }}^{\prime}\right)=I\left(A_{k} B_{k}: A_{s} B_{s}\right)_{\gamma_{\text {Bell }}}=H\left(\left\{p_{k}\right\}\right)
\end{aligned}
$$

where $I\left(A_{k} B_{k}: A_{s} B_{s}\right)$ is the quantum mutual information and $H\left(\left\{p_{k}\right\}\right)$ is the entropy of $\left\{p_{k}\right\}$. Since $\gamma_{\text {Bell }}$ commutes with both $\hat{\gamma}_{\text {Bell }}$ and $\dot{\gamma}_{\text {Bell }}$, these values are achieved also by performing a global measurement first and then computing the classical relative entropies [24, 34]. In both cases the optimal measurement operators are $\left\{M_{a b j}\right\}=\left\{P_{\phi_{a b}} \otimes P_{\sigma_{j}}\right\}$.

Another simplification of Bell private states with respect to general private states involves the expression for the distillable entanglement in $A_{k} B_{k}$ :

Lemma 8. For all Bell private states $\gamma_{\text {Bell }}^{m}$ it holds

$$
E_{D}\left(\gamma_{B e l l, A_{k} B_{k}}^{m}\right)=m-H\left(\left\{p_{k}\right\}\right)
$$

where $\gamma_{\text {Bell }, A_{k} B_{k}}^{m}=\operatorname{tr}_{A_{s} B_{s}} \gamma_{\text {Bell }}^{m}$.
Proof. For all private states, the reduced state of $A_{k} B_{k}$ has support only on the maximally correlated subspace. For such reduced states, it has been shown [31] that the distillable entanglement is equal to the hashing bound [10]:

$$
E_{D}\left(\operatorname{tr}_{A_{s} B_{s}} \gamma\right)=H\left(B_{k}\right)_{\gamma}-H\left(A_{k} B_{k}\right)_{\gamma} .
$$

For Bell private states we have

$$
\operatorname{tr}_{A_{s} B_{s}} \gamma_{B e l l}^{m}=\sum_{j} p_{j} \phi_{0 j}
$$

The marginal of $B_{k}$ will be completely mixed so $H\left(B_{k}\right)_{\gamma}$ will be maximal, while $H\left(A_{k} B_{k}\right)_{\gamma}$ is the entropy of $\left\{p_{k}\right\}$ :

$$
E_{D}\left(\operatorname{tr}_{A_{s} B_{s}} \gamma_{\text {Bell }}^{m}\right)=m-H\left(\left\{p_{k}\right\}\right) .
$$

Of particular interest is the case of uniform $\left\{p_{k}\right\}$, then:

$$
E_{D}\left(\operatorname{tr}_{A_{s} B_{s}} \gamma_{B e l l}\right)=0
$$

which is independent of the key systems size.

\section{Appendix C: Relative Entropies}

The quantum relative entropy between two states $\rho$ and $\sigma$ is defined as:

$$
D(\rho \| \sigma):=\operatorname{tr} \rho[\log \rho-\log \sigma] .
$$

Some interesting entanglement measures are defined using the relative entropy.

\section{Definition 9 ([25], Relative entropy of entangle- ment).}

$$
E_{R}(\rho):=\inf _{\sigma \in S E P} D(\rho \| \sigma)
$$

This was generalized as follows.

Definition 10 ([11], Relative entropy with respect to $\boldsymbol{P})$. Let $P$ be any set of states $P$ containing at least one full rank state. Define:

$$
E_{R}^{P}(\rho):=\inf _{\sigma \in P} D(\rho \| \sigma) .
$$

By taking the relative entropy with respect to separable states, we recover the relative entropy of entanglement. The relative entropy with respect to $P$ is asymptotically continuous [27], a useful property needed to prove our bounds on rates. We will restate asymptotic continuity here in the form we need it, using the improved bounds from [33, Lemma 7].

Lemma 11 ([33]). Let $C=\sup _{\rho} E_{R}^{P}(\rho)$. Then:

$$
\left|E_{R}^{P}(\rho)-E_{R}^{P}(\sigma)\right| \leq C \varepsilon-\eta(\varepsilon)+\eta(1+\varepsilon)
$$

where $\varepsilon=\frac{1}{2}\|\rho-\sigma\|_{1}$ and $\eta(x)=x \log x$. 
In particular for $P \supseteq S E P_{A: B}$ and $d=\min \{|A|,|B|\}$, we can always take $C=\log d$.

For an arbitrary set of measurements $\mathbb{M}$ we define the M-relative entropy [11]. However, for now we allow any set of quantum maps $\mathbb{L}$ (completely positive trace preserving maps) in the definition, as opposed to [11] where $\mathbb{L}$ only contains measurements.

Definition 12 ([11], $\mathbb{L}$-relative entropy). Let $\mathbb{L}$ be any set of quantum maps. Define

$$
D_{\mathbb{L}}(\rho \| \sigma)=\sup _{\Lambda \in \mathbb{L}} D(\Lambda(\rho) \| \Lambda(\sigma)) .
$$

Originally the above relative entropy was defined only for sets of measurements $\mathbb{M}$ However, for now we will allow any set of quantum maps (completely positive trace preserving maps) in the definition, as opposed to [11] where $\mathbb{L}$ only contains measurements. Combining Definitions 10 and 12 we obtain the following definition.

Definition 13 ([11], $\mathbb{L}$-relative entropy with respect to $P)$. Let $\mathbb{L}$ be any set of quantum maps and let $P$ be any set of states $P$ containing at least one full rank state. Define

$$
E_{R, \mathbb{L}}^{P}(\rho)=\inf _{\sigma \in P} D_{\mathbb{L}}(\rho \| \sigma) .
$$

\section{Appendix D: Regularized relative entropies}

Given a function on states $f$ we can consider the standard regularization

$$
f^{\infty}(\rho)=\lim _{n \rightarrow \infty} \frac{1}{n} f\left(\rho^{\otimes n}\right)
$$

whenever the limit is well defined. Similarly for functions $g$ of two states we can consider a regularization

$$
g^{\infty}(\rho, \sigma)=\lim _{n \rightarrow \infty} \frac{1}{n} g\left(\rho^{\otimes n}, \sigma^{\otimes n}\right)
$$

again whenever the limit is well defined.

Fekete's lemma [12] guarantees that the regularization will be well defined, at least for the classes of partial measurements that we consider in Theorem 2 and Corollary 25. We call a sequence $D_{n}$ super-additive if it satisfies

$$
\forall n, m: D_{m+n} \geq D_{n}+D_{m} .
$$

The lemma states that if a sequence $D_{n}$ is super-additive, then $\frac{1}{n} D_{n}$ either converges or diverges to infinity, more specifically $\lim _{n \rightarrow \infty} \frac{1}{n} D_{n}=\sup _{n \in \mathbb{N}} \frac{1}{n} D_{n}$.

Fekete's lemma guarantees that we can regularize the various relative entropies defined above, as long as $\mathbb{L}$ and $P$ make these relative entropies super-additive. This is the case for classes of maps like $L O C C$ that are defined for states $\rho$ and $\rho^{\otimes n}$ alike, and that map separable states to separable state. To make this rigorous we need to define $\mathbb{L}$ and $P$ for every $n$ and therefore we need to explicitly consider the Hilbert space $\mathcal{H}$ and the set of density matrices $\mathcal{D}(\mathcal{H})$ for $n=1$; let $\mathcal{H}$ be finite dimensional.

We say that $\mathbb{L}$ is a class closed under tensor products, or simply class, of quantum maps (or measurements) if it is a sequence of $\mathbb{L}_{n}$, each a set of quantum maps on $\mathcal{D}\left(\mathcal{H}^{\otimes n}\right)$, such that:

$$
\forall i, j, \Lambda \in \mathbb{L}_{i}, \Gamma \in \mathbb{L}_{j}: \quad \Lambda \otimes \Gamma \in \mathbb{L}_{i+j} .
$$

In such case it is easy to check that $D_{\mathbb{L}_{n}}\left(\rho^{\otimes n} \| \sigma^{\otimes n}\right)$ satisfies Equation (D1),

$$
D_{\mathbb{L}_{i+j}}\left(\rho^{\otimes i+j} \| \sigma^{\otimes i+j}\right) \geq D_{\mathbb{L}_{i}}\left(\rho^{\otimes i} \| \sigma^{\otimes i}\right)+D_{\mathbb{L}_{j}}\left(\rho^{\otimes j} \| \sigma^{\otimes j}\right),
$$

and thus we can define the following regularization.

Definition 14 (Regularized $\mathbb{L}$-relative entropy). Let $\mathbb{L}$ be a class of quantum maps closed under tensor products. Then, define:

$$
D_{\mathbb{L}}^{\infty}(\rho \| \sigma)=\lim _{n \rightarrow \infty} \frac{1}{n} D_{\mathbb{L}_{n}}\left(\rho^{\otimes n} \| \sigma^{\otimes n}\right) .
$$

We now restrict $\mathbb{L}$ to be a class of $\mathbb{M}$ measurements only, so that we can regularize $D_{\mathbb{M}}^{P}$. As done in [11], we also need to impose further conditions on $P$ with respect to $\mathbb{M}$ and on $P$ itself.

We say that $P$ is a class closed under partial trace, or simply class, of states, if it is a sequence of $P_{n}$, each a convex set of states in $\mathcal{D}\left(\mathcal{H}^{\otimes n}\right)$, such that for all $n$ :

$$
\sigma \in P_{n} \quad \Rightarrow \quad\left(\operatorname{tr}_{I} \sigma \in P_{n-|I|} \quad \forall I \subset\{1 \ldots n\}\right)
$$

where $\operatorname{tr}_{I}$ is the partial trace over the systems designated by the index set $I$. Furthermore, let $\mathbb{M}$ be a class of measurements, we say that a class of states $P$ is closed under $\mathbb{M}$, if for all $n>m$, it holds that

$$
\left\{\begin{array}{l}
\sigma \in P_{n} \\
\mathcal{M} \in \mathbb{M}_{m}
\end{array} \quad \Rightarrow \quad \frac{\operatorname{tr}_{I} M_{k}^{I} \sigma}{\operatorname{tr} M_{k}^{I} \sigma} \in P_{n-m} \quad \forall k,|I|=m\right.
$$

where $\left\{M_{k}\right\}$ are the measurements operators of $\mathcal{M}$ and $M_{k}^{I}$ are the operators acting on systems $I\left(M_{k}^{I} \equiv M_{k}^{I} \otimes \mathbb{1}\right.$, where the identity acts on the complement of $I$ ). These properties are enough to guarantee that the $\mathcal{M}$-relative entropy with respect to $P$ also satisfies Equation (D1). While the original statement only considers separable states, PPT states, separable measurements and LOCC measurements, the same exact proof carries over to general $P$ and $\mathbb{M}$

Lemma 15 ([11, Theorem 2(d)]). Let $\mathbb{M}$ be a class of measurements and let $P$ be a class of states closed under M. Then:

$$
E_{R, \mathbb{M}_{m+n}}^{P}\left(\rho^{\otimes m+n}\right) \geq E_{R, \mathbb{M}_{m}}^{P}\left(\rho^{\otimes m}\right)+E_{R, \mathbb{M}_{n}}^{P}\left(\rho^{\otimes n}\right) .
$$

Thanks to this lemma the following regularization is now well defined. 


\begin{tabular}{|c|c|c|}
\hline \multicolumn{2}{|l|}{ Notation } & \multirow[t]{2}{*}{ Meaning } \\
\hline Full & Short & \\
\hline$A_{k} B_{k}$ & & The key systems of Alice and Bob, $\left|A_{k}\right|=\left|B_{k}\right|$. We define $m=\log _{2}\left|A_{k}\right|$. \\
\hline$A_{s} B_{s}$ & & The shield systems of Alice and Bob. In most the examples we use $\left|A_{s}\right|=\left|B_{s}\right|=d$. \\
\hline$\rho, \hat{\rho}$ & & A state on $A_{k} B_{k} A_{s} B_{s}$ and its key attacked state \\
\hline $\mathcal{M}$ & & A measurement. \\
\hline$\Lambda$ & & A map/protocol. \\
\hline$L O_{A}, L O_{B}$ & & Local operations: any quantum channel acting only on Alice's or Bob's systems. \\
\hline $\begin{array}{l}L O C C_{A: B} \\
L O C C_{A \rightarrow B}\end{array}$ & & Local Operations with two-way and one-way Classical Communication. \\
\hline $\mathcal{M} \in L O_{A}$ & $M_{A}$ & A measurement at Alice's side. \\
\hline $\begin{array}{l}\mathcal{M} \in L O C C_{A: B} \\
\mathcal{M} \in L O C C_{A \rightarrow B}\end{array}$ & $\begin{array}{l}\mathcal{M}_{A: B} \\
\mathcal{M}_{A \rightarrow B} \\
\end{array}$ & A measurement in $L O C C_{A: B}$ and $L O C C_{A: B}$ respectively. \\
\hline $\mathcal{M} \in L O C C_{\mathbb{M} A: B}$ & $\mathcal{M}_{\mathbb{M} A: B}$ & A partial measurement in $L O C C_{A: B}$, Alice needs to measure, but not Bob. \\
\hline $\begin{array}{l}\Lambda \in L O C C_{A: B} \\
\Lambda \in L O C C_{A \rightarrow B}\end{array}$ & $\begin{array}{l}\Lambda_{A: B} \\
\Lambda_{A \rightarrow B}\end{array}$ & A protocol in $L O C C_{A: B}$ and $L O C C_{A \rightarrow B}$ respectively \\
\hline$D_{A}$ & & Relative entropy restricted to partial measurements $\mathbb{L}=\mathbb{M} \otimes \mathrm{id}_{A}$, where $\mathbb{M}=L O_{A}$. \\
\hline$D_{A \rightarrow B}$ & & Relative entropy restricted to measurements in $\mathbb{M}=L O C C_{A \rightarrow B}$ \\
\hline$E_{R, A \rightarrow B}$ & & Relative entropy with measurements in $\mathbb{M}=L O C C_{A \rightarrow B}$ and $P=S E P_{A: B}$ \\
\hline
\end{tabular}

TABLE 6. General notation for states, maps, measurements, parties, etc.

\begin{tabular}{|c|c|c|}
\hline \multicolumn{2}{|c|}{ Repeater notation } & \multirow[t]{2}{*}{ Meaning } \\
\hline Full & Short & \\
\hline$C C^{\prime}$ & $C$ & The parties on Charlie's side. $C$ and $C^{\prime}$ share entanglement with Alice and Bob respectively. \\
\hline$C_{k} C_{k}^{\prime}$ & $C_{k}$ & The key systems of Charlie's parties. \\
\hline$C_{s} C_{s}^{\prime}$ & $C_{s}$ & The shield systems of Charlie's parties. \\
\hline$\sigma \in S E P_{A: C: C^{\prime}: B}$ & $\sigma_{A: C: C^{\prime}: B}$ & Quadri-separable states of Alice, Bob, and Charlie's parties. \\
\hline$\sigma \in S E P_{A: C B}$ & $\sigma_{A: C B}$ & Separable states between Alice and the joint systems of Charlie and Bob. \\
\hline$\sigma \in S E P_{A C: B}$ & $\sigma_{A C: B}$ & Separable states between Bob and the joint systems of Charlie and Alice. \\
\hline$L O C C_{A: C: B}$ & & Tripartite LOCC of Alice, Charlie and Bob. \\
\hline$L O C C_{C \rightarrow A B}$ & & $\begin{array}{l}\text { Tripartite LOCC with only one-way communication from Charlie to Alice/Bob. Alice and } \\
\text { Bob can communicate freely with each other. }\end{array}$ \\
\hline $\mathcal{M} \in L O_{C}$ & $\mathcal{M}_{C}$ & A partial measurement at Charlie's. \\
\hline $\begin{array}{l}\mathcal{M} \in L O C C_{A: C: B} \\
\mathcal{M} \in L O C C_{C \rightarrow A B}\end{array}$ & $\begin{array}{l}\mathcal{M}_{A: C: B} \\
\mathcal{M}_{C \rightarrow A B}\end{array}$ & A measurement in $L O C C_{A: C: B}$ and $L O C C_{C \rightarrow A B}$ respectively. \\
\hline $\begin{array}{l}\Lambda \in L O C C_{A: C: B} \\
\Lambda \in L O C C_{C \rightarrow A B}\end{array}$ & $\begin{array}{l}\Lambda_{A: C: B} \\
\Lambda_{C \rightarrow A B}\end{array}$ & A protocol in $L O C C_{A: C: B}$ and $L O C C_{C \rightarrow A B}$ respectively. \\
\hline$D_{C}$ & & Relative entropy restricted to partial measurements $\mathbb{L}=\mathbb{M} \otimes \mathrm{id}_{A B}$, where $\mathbb{M}=L O_{C}$. \\
\hline$D_{C \rightarrow A B}$ & & Relative entropy restricted to measurements in $\mathbb{M}=L O C C_{C \rightarrow A B}$ \\
\hline$E_{R, C \rightarrow C^{\prime}: B}^{A: C}$ & & Relative entropy with $P=S E P_{A: C: C^{\prime}: B}$ and measurements in $\mathbb{M}=L O C C_{C \rightarrow A B}$. \\
\hline $\begin{array}{l}E_{R: \boldsymbol{C}}^{A: \boldsymbol{B}} \rightarrow \underset{B}{\rightarrow} A B \\
E_{R, \boldsymbol{C} \rightarrow A B}^{A C \rightarrow}\end{array}$ & & Relative entropy with $P=S E P_{A: C B}, S E P_{A C: B}$ and measurements in $\mathbb{M}=L O C C_{C \rightarrow A B}$. \\
\hline $\begin{array}{l}E_{R, \mathbb{M} B}^{A: \boldsymbol{C}}: A B \\
E_{R, \mathbb{M} \boldsymbol{C}: A B}^{A: C B}\end{array}$ & & $\begin{array}{l}\text { Relative entropy with } P=S E P_{A: C B}, S E P_{A C: B} \text { and } \mathbb{L}=L O C C_{\mathbb{M} \boldsymbol{C} \rightarrow A B} \text {, which are LOCC } \\
\text { protocols of } \boldsymbol{C}: A B \text { followed by a measurement at Charlie. }\end{array}$ \\
\hline
\end{tabular}

TABLE 7. Additional notation regarding the repeater setting. 


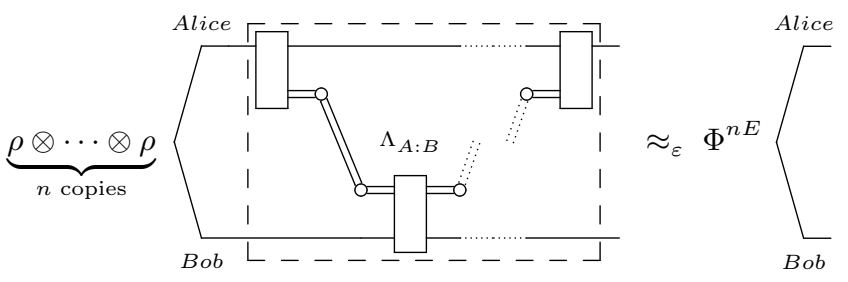

FIG. 8. Quantum circuit for entanglement distillation. The circuit in the dashed box is the map $\Lambda \in L O C C_{A: B}$, optimized to output $\Phi^{n E}$ with the highest $n E$ possible.

Definition 16 (Regularized M-relative entropy with respect to $P)$. Let $\mathbb{M}$ be a class of measurements and let $P$ be a class of states closed under $\mathbb{M}$. Define:

$$
E_{R, \mathbb{M}}^{\infty, P}(\rho)=\lim _{n \rightarrow \infty} \frac{1}{n} E_{R, \mathbb{M}}^{P}\left(\rho^{\otimes n}\right) .
$$

Notice that the class of PPT states is closed under the class of PPT measurements and consequently under all subclasses of measurements, like separable and LOCC measurements. Similarly the class of separable states is closed under the class of separable measurements, and consequently under LOCC measurements. Thus Definition 16 is always well defined for the above combinations of states and measurements.

As it is usually done, we will omit the fact that the classes of states and quantum maps/measurements are actually sequences in such regularized quantities, therefore we will drop the index $n$. We will use $\sigma_{P}$ and $\mathcal{M}_{\mathbb{M}}$ as short hand notation for $\sigma \in P$ and $\mathcal{M} \in \mathbb{M}$, respectively. See Table 6 for a more detailed list of symbols and notations.

Finally, we highlight that we do not have yet a general Definition 16 for arbitrary classes of quantum maps. This in particular includes classes of partial measurements, where only some parties are forced to measure their systems. Already for partial measurements, the only regularized definition that we can use for now is Definition 14.

\section{Appendix E: Entanglement and Key distillation}

When performing distillation, the goal is to approximate a desired output state by acting on the input via the allowed operations. Namely, four elements define a rate: the input state, the target states, the allowed operations and a measure of approximation. Beyond this, all the rates have a common structure. Usually, trace norm distance is used to quantify the approximation between two states. We will use the notation

$$
\varrho \approx_{\varepsilon} \varsigma \quad \Leftrightarrow \quad \frac{1}{2}\|\varrho-\varsigma\|_{1} \leq \varepsilon .
$$

In what follows we will use the same convention as in [4] in the definitions of rates, namely all rates are defined as a single formula.
The distillable entanglement of a state $\rho$ is then formally defined as the rate at which maximally entangled states can be distilled under bipartite LOCC [20]:

$$
E_{D}\left(\rho_{A B}\right)=\lim _{\varepsilon \rightarrow 0} \lim _{n \rightarrow \infty} \sup _{\Lambda_{A: B}}\left\{E: \Lambda\left(\rho_{A B}^{\otimes n}\right) \approx_{\varepsilon} \Phi_{A B}^{n E}\right\}
$$

as illustrated in Figure 8.

The distillable key is defined as the rate at which perfect secret bits can be distilled. However it also equals the rate at which private states can be distilled under bipartite LOCC [2]:

$$
K_{D}(\rho)=\lim _{\varepsilon \rightarrow 0} \lim _{n \rightarrow \infty} \sup _{\Lambda_{A: B}}\left\{K: \Lambda\left(\rho^{\otimes n}\right) \approx_{\varepsilon} \gamma^{n K}\right\} .
$$

Note how the expression is almost the same as for distillable entanglement, the only difference are the desired output states. Using the reversible map of Lemma 1 it is now also possible to write the distillable key as the rate at which Bell private states can be distilled:

$$
K_{D}(\rho)=\lim _{\varepsilon \rightarrow 0} \lim _{n \rightarrow \infty} \sup _{\Lambda_{A: B}}\left\{K: \Lambda\left(\rho^{\otimes n}\right) \approx_{\varepsilon} \gamma_{\text {Bell }}^{n K}\right\} .
$$

While the target state defines the kind of resource being measured (pure entanglement, key, ...), changing the available protocols produces variations of these quantities that reflect different scenarios, like in the case of the one-way distillable entanglement. This is:

$$
E_{D}(\rho)=\lim _{\varepsilon \rightarrow 0} \lim _{n \rightarrow \infty} \sup _{\Lambda_{A \rightarrow B}}\left\{E: \Lambda\left(\rho^{\otimes n}\right) \approx_{\varepsilon} \Phi^{n E}\right\}
$$

where the maps are restricted to one-way $L O C C$. Just like there is a regularized $L O C C$ restricted relative entropy of entanglement upper bound on $E_{D}$, the same proof carries over to $E_{D}$ by simply restricting to one-way $L O C C$. Here below, we give an explicit proof of such bound.

Lemma 17 ([14]). For any state $\rho$ and any separable state $\sigma$, it holds:

$$
D_{A}^{\infty}(\rho \| \sigma) \geq E_{R, A \rightarrow B}^{\infty}(\rho) \geq E_{D}(\rho) .
$$

It should be understood that the direction of the communication in the measurement must be the same as the direction in the distillation protocol.

Proof. The proof of $E_{R, A \rightarrow B}^{\infty}(\rho) \geq E_{D}(\rho)$ mirrors the same result for two-way LOCC from [14]. We need two results from the same article, the value of $E_{R, A \rightarrow B}$ on maximally entangled states [14, Proposition 4]:

$$
\begin{aligned}
E_{R, A \rightarrow B}\left(\Phi^{m}\right) & =\log \left(2^{m}+1\right)-1 \\
& =m+\log \left(1+2^{-m}\right)-1,
\end{aligned}
$$

and its asymptotic continuity [14, Proposition 3]:

$$
\left|E_{R, A \rightarrow B}(\varrho)-E_{R, A \rightarrow B}(\varsigma)\right| \leq 4 \varepsilon \log \frac{3|A B|}{\varepsilon}
$$


for states satisfying $\frac{1}{2}\|\varrho-\varsigma\|_{1}=\varepsilon \leq e^{-1}$. Now, for all $\varepsilon$ and for all $n$ let $\Lambda$ be the optimal one-way distillation map of $E_{D}(\rho)$. Then:

$$
\begin{aligned}
& \frac{1}{n} E_{R, A \rightarrow B}\left(\rho^{\otimes n}\right) \\
& \quad \geq \frac{1}{n} E_{R, A \rightarrow B}\left(\Lambda\left(\rho^{\otimes n}\right)\right) \\
& \quad \geq \frac{1}{n} E_{R, A \rightarrow B}\left(\Phi^{n E}\right)-\frac{1}{n} 4 \varepsilon \log \frac{32^{2 n E}}{\varepsilon} \\
& \quad=E+\frac{1}{n} \log \left(1+\frac{1}{2^{n E}}\right)-\frac{1}{n}-8 \varepsilon E-\frac{4 \varepsilon}{n} \log \frac{3}{\varepsilon}
\end{aligned}
$$

Taking the limit $n \rightarrow \infty$ leads to:

$$
E_{R, A \rightarrow B}^{\infty}(\rho) \geq E-8 \varepsilon E
$$

and taking the limit $\varepsilon \rightarrow 0$ ends the first part of the proof.

We now prove $D_{A}^{\infty}(\rho \| \sigma) \geq D_{A \rightarrow B}^{\infty}(\rho \| \sigma)$. This follows because the optimization in $D_{A}$ is made over a larger class of maps, because Bob does not necessarily measure. More precisely, any measurement in $\mathcal{M} \in L O C C_{A \rightarrow B}$, can always be written as a measurement $\mathcal{M}^{\prime} \in L O_{A}$ followed by a measurement on Bob conditioned on Alice's outcome [10], namely a global measurement $\mathcal{M}^{\prime \prime}$ acting on Bob and Alice's measurement outcome. The communication is implicit in the fact that $\mathcal{M}^{\prime \prime}$ has received the outcome of $\mathcal{M}^{\prime}$ and is treating $\mathcal{M}^{\prime}(\rho)$ as a global state. By the monotonicity of the relative entropy we thus have:

$$
\begin{aligned}
D\left(\mathcal{M}^{\prime}(\rho) \| \mathcal{M}^{\prime}(\sigma)\right) & \geq D\left(\mathcal{M}^{\prime \prime} \circ \mathcal{M}^{\prime}(\rho) \| \mathcal{M}^{\prime \prime} \circ \mathcal{M}^{\prime}(\sigma)\right) \\
& =D(\mathcal{M}(\rho) \| \mathcal{M}(\sigma))
\end{aligned}
$$

Thus:

$$
D_{A}(\rho \| \sigma) \geq D_{A \rightarrow B}(\rho \| \sigma)
$$

and therefore

$$
D_{A}^{\infty}(\rho \| \sigma) \geq D_{A \rightarrow B}^{\infty}(\rho \| \sigma) .
$$

Since by definition $D_{A \rightarrow B}^{\infty}(\rho \| \sigma) \geq E_{R, A \rightarrow B}^{\infty}(\rho)$, this concludes the proof.

\section{Appendix F: Distillable entanglement of private states}

In this Appendix we provide a rigorous proof of Theorem 2. We start by proving the in-line claims of the main text.

Lemma 18. Let $\rho$ be a key correlated state of the form $\rho=\frac{1}{2^{m}} \sum_{j} \phi_{0 j} \otimes \sigma_{j}$. Then it holds:

$$
E_{D}^{\rightarrow}(\rho) \geq \sup _{\mathcal{M} \in L O_{A}} \frac{1}{2^{m}} \sum_{j} D\left(\mathcal{M}\left(\sigma_{j}\right) \| \mathcal{M}(\sigma)\right)
$$

where $\sigma=\sum_{j} \frac{1}{2^{m}} \sigma_{j}$,
Proof. Let Alice perform a measurement $\mathcal{M}: A_{s} \rightarrow M$ on her shield and define:

$$
\tilde{\rho}:=\frac{1}{\left|B_{k}\right|} \sum_{j} \phi_{0 j} \otimes \mathcal{M}\left(\sigma_{j}\right) .
$$

We have $E_{D} \rightarrow(\rho) \geq E_{D}(\tilde{\rho})$. Alice now sends the outcome to Bob and then by the hashing bound [10] we find:

$$
E_{D}^{\rightarrow}(\tilde{\rho}) \geq\left[H\left(B_{k} B_{s} M\right)_{\tilde{\rho}}-H\left(A_{k} B_{k} B_{s} M\right)_{\tilde{\rho}}\right],
$$

where $H(S)_{\rho}:=-\operatorname{tr}\left[\rho_{S} \log \rho_{S}\right]$ is the quantum entropy of $\rho_{S}$ on system $S$. However, because the key systems are in a mixture of Bell states, tracing out $A_{k}$ will leave $B_{k}$ in product with $B_{s} M$. Furthermore, the mixture of Bell states is uniform and thus $H\left(B_{k}\right)_{\tilde{\rho}}=H\left(A_{k} B_{k}\right)_{\tilde{\rho}}$, therefore:

$$
H\left(B_{k} B_{s} M\right)_{\tilde{\rho}}=H\left(A_{k} B_{k}\right)_{\tilde{\rho}}+H\left(B_{s} M\right)_{\tilde{\rho}}
$$

We now use that

$$
H(X)_{\alpha}+H(Y)_{\alpha}-H(X Y)_{\alpha}=D\left(\alpha_{X Y} \| \alpha_{X} \otimes \alpha_{Y}\right)
$$

(where $\alpha$ is a state on $X Y$ ) and conclude:

$$
\begin{aligned}
E_{D}^{\rightarrow}(\rho) & \geq\left[H\left(A_{k} B_{k}\right)_{\tilde{\rho}}+H\left(B_{s} M\right)_{\tilde{\rho}}-H\left(A_{k} B_{k} B_{s} M\right)_{\tilde{\rho}}\right] \\
& =D\left(\frac{1}{\left|B_{k}\right|} \sum_{j} \phi_{0 j} \otimes \mathcal{M}\left(\sigma_{j}\right) \| \frac{1}{\left|B_{k}\right|} \sum_{j} \phi_{0 j} \otimes \mathcal{M}(\sigma)\right) \\
& =\frac{1}{\left|B_{k}\right|} \sum_{j} D\left(\mathcal{M}\left(\sigma_{j}\right) \| \mathcal{M}(\sigma)\right)
\end{aligned}
$$

where $\sigma=\frac{1}{\left|B_{k}\right|} \sum_{j} \sigma_{j}$. Taking the supremum over $\mathcal{M}$ proves the claim.

Corollary 19. For any key correlated state $\rho$, it holds:

$$
E_{D}(\rho) \geq \sup _{\mathcal{M} \in L O_{A}} \frac{1}{2^{m}} \sum_{j} D\left(\mathcal{M}\left(\rho_{j}\right) \| \mathcal{M}(\hat{\rho})\right)
$$

where $\rho_{j}:=\mathcal{Z}_{B_{k}}^{j}(\rho)$.

Proof. We can use Lemma 18 after using Lemma 1:

$$
\begin{aligned}
E_{D}^{\rightarrow}(\rho) & =E_{D}^{\rightarrow}(\mathcal{E}(\rho))=E_{D}^{\rightarrow}\left(\sum_{j} \frac{1}{\left|B_{k}\right|} \phi_{0 j} \otimes \rho_{j}\right) \\
& \geq \sup _{\mathcal{M} \in L O_{A}} \frac{1}{\left|B_{k}\right|} \sum_{j} D\left(\mathcal{M}\left(\rho_{j}\right) \| \mathcal{M}(\hat{\rho})\right)
\end{aligned}
$$

where we used that $\hat{\rho}=\frac{1}{\left|B_{k}\right|} \sum_{j} \rho_{j}$.

These bounds generalize to the two-way distillable entanglement because we can always apply Corollary 19 after a two-way preprocessing as shown below.

Corollary 20. For any key correlated state $\rho$, it holds:

$$
E_{D}(\rho) \geq \sup _{\mathcal{M}_{\mathbb{M} A: B}} \frac{1}{2^{m}} \sum_{j} D\left(\mathcal{M}\left(\rho_{j}\right) \| \mathcal{M}(\hat{\rho})\right)
$$

where $L O C C_{\mathbb{M} A: B}$ are all two-way LOCC protocol that ends with a measurement at Alice's. 
Proof. By Lemma 1:

$$
\begin{aligned}
E_{D}(\rho) & =E_{D}(\mathcal{E}(\rho)) \\
& =E_{D}\left(\sum_{j} \frac{1}{2^{m}} \phi_{0 j} \otimes \rho_{j}\right) \\
& \geq \sup _{\Lambda} E_{D}^{\rightarrow}\left(\sum_{j} \frac{1}{2^{m}} \phi_{0 j} \otimes \Lambda\left(\rho_{j}\right)\right)
\end{aligned}
$$

where $\Lambda$ are arbitrary LOCC protocols. We then use Lemma 18:

$$
E_{D}(\rho) \geq \sup _{\Lambda} \sup _{\mathcal{M}^{\prime}} \frac{1}{2^{m}} \sum_{j} D\left(\mathcal{M}^{\prime} \circ \Lambda\left(\rho_{j}\right) \| \mathcal{M}^{\prime} \circ \Lambda(\hat{\rho})\right)
$$

however without loss of generality we can write any measurement in $L O C C_{A: B}$ as $\mathcal{M}^{\prime} \circ \Lambda$, where $\mathcal{M}^{\prime}$ is a measurement at Alice's and $\Lambda$ an $L O C C_{A: B}$ protocol, therefore

$$
E_{D}(\rho)=\sup _{\mathcal{M}_{\mathbb{M} A: B}} \frac{1}{2^{m}} \sum_{j} D\left(\mathcal{M}\left(\rho_{j}\right) \| \mathcal{M}(\hat{\rho})\right) .
$$

We can finally give a rigorous proof of the main theorem.

Theorem 21 (Main text Theorem 2). For any key correlated state $\rho$, it holds:

$$
\begin{aligned}
& E_{D}^{\vec{D}}(\rho) \geq D_{A}(\rho \| \hat{\rho}):=\sup _{\mathcal{M} \in L O_{A}} D(\mathcal{M}(\rho) \| \mathcal{M}(\hat{\rho})) \\
& E_{D}^{\rightarrow}(\rho) \geq D_{A}^{\infty}(\rho \| \hat{\rho}):=\lim _{n \rightarrow \infty} \frac{1}{n} D_{A}\left(\rho^{\otimes n} \| \hat{\rho}^{\otimes n}\right)
\end{aligned}
$$

If $\hat{\rho}$ is also separable then:

$$
E_{D}^{\rightarrow}(\rho)=D_{A}^{\infty}(\rho \| \hat{\rho})
$$

Proof. It is straightforward to check that $\mathcal{Z}_{B_{k}}^{j}(\hat{\rho})=\hat{\rho}$. Let $\mathcal{M}$ be any measurement $\mathcal{M}$ at Alice. Because the measurement is local at Alice, it commutes with the unitary $\mathcal{Z}^{j}$ at Bob, thus we have:

$$
\begin{aligned}
D\left(\mathcal{M}\left(\rho_{j}\right) \| \mathcal{M}(\hat{\rho})\right) & =D\left(\mathcal{M} \otimes \mathcal{Z}_{B_{k}}^{j}(\rho) \| \mathcal{M} \otimes \mathcal{Z}_{B_{k}}^{j}(\hat{\rho})\right) \\
& =D(\mathcal{M}(\rho) \| \mathcal{M}(\hat{\rho}))
\end{aligned}
$$

where that the relative entropy is unitary invariant. We can now rewrite Corollary 19 as:

$$
\begin{aligned}
E_{D}^{\vec{D}}(\rho) & \geq \sup _{\mathcal{M} \in L O_{A}} \frac{1}{2^{m}} \sum D\left(\mathcal{M}\left(\rho_{j}\right) \| \mathcal{M}(\hat{\rho})\right) \\
& =\sup _{\mathcal{M} \in L O_{A}} \frac{1}{2^{m}} \sum D(\mathcal{M}(\rho) \| \mathcal{M}(\hat{\rho})) \\
& =\sup _{\mathcal{M} \in L O_{A}} D(\mathcal{M}(\rho) \| \mathcal{M}(\hat{\rho}))
\end{aligned}
$$

proving Equation (F6). For Equation (F7) we have:

$$
E_{D}^{\rightarrow}(\rho)=\frac{1}{n} E_{D}^{\rightarrow}\left(\rho^{\otimes n}\right) \geq \frac{1}{n} D_{A}\left(\rho^{\otimes n} \| \hat{\rho}^{\otimes n}\right) \quad \forall n
$$

because the distillable entanglement is already regularized and $\rho^{\otimes n}$ is still a key correlated state. By Fekete's Lemma [12], $\frac{1}{n} D_{A}\left(\rho^{\otimes n} \| \hat{\rho}^{\otimes n}\right)$ converges because $D_{A}$ is super-additive; taking the limit $n \rightarrow \infty$ proves Equation (F7). Equality in Equation (F8) follows because if $\hat{\rho}$ is separable, then we get the opposite inequality from Lemma 17:

$$
D_{A}^{\infty}(\rho \| \hat{\rho}) \geq E_{D}^{\rightarrow}(\rho) \geq D_{A}^{\infty}(\rho \| \hat{\rho})
$$

We now show with an example that using the reversible map is often necessary. We can provide an example of a Bell private state for which the bound of Lemma 18 is strictly suboptimal, while the bound of Corollary 19 achieves equality.

Example 22. Let $\left|A_{k}\right|=\left|B_{k}\right|=\left|A_{s}\right|^{2}=\left|B_{s}\right|^{2}=2^{2 m}$, namely the key systems have now $2 m$ qubits each while the shield have only $m$ qubits each. For the following example we need to use the whole Bell basis. We define the private state:

$$
\begin{aligned}
& \gamma^{2 m}=\sum_{i j} \frac{1}{2^{2 m}} \phi_{0 i} \otimes \phi_{0 j} \otimes \phi_{i j} \\
& \hat{\gamma}^{2 m}=\hat{\Phi}^{m} \otimes \hat{\Phi}^{m} \otimes \tau^{m}
\end{aligned}
$$

where $\tau^{m}=\frac{\mathbb{1}}{2^{2 m}}=\sum_{i j} \frac{1}{2^{2 m}} \phi_{i j}$ is the maximally mixed state of the shield. By the optimality result of [29, Equation 8], the bound of Lemma 18 computes to:

$$
\sup _{\mathcal{M}_{A}} \frac{1}{2^{2 m}} \sum_{i j} D\left(\mathcal{M}\left(\phi_{i j}\right) \| \mathcal{M}\left(\tau^{m}\right)\right)=m .
$$

which is achieved measuring the computational basis. However, this state is distillable into $2 m$ maximally entangled states with just a sequence of unitaries. When changing from the computational basis to the conjugate basis on both sides (bilaterally), $\phi_{i j}$ converts to $\phi_{j i}$ and recall that $B N O T \phi_{0 j} \otimes \phi_{k l} B N O T^{\dagger}=\phi_{0, j-l} \otimes \phi_{k l}$ (Lemma 5). The unitary that distills $2 m$ maximally entangled states is then obtained by the following sequence:

- Applying BNOT to the second and third Bell states results in:

$$
\gamma^{2 m} \rightarrow \sum_{i j} \frac{1}{2^{2 m}} \phi_{0 i} \otimes \phi_{00} \otimes \phi_{i j}
$$

- Applying the bilateral change of basis on the third Bell state results in:

$$
\sum_{i j} \frac{1}{2^{2 m}} \phi_{0 i} \otimes \phi_{00} \otimes \phi_{i j} \rightarrow \sum_{i j} \frac{1}{2^{2 m}} \phi_{0 i} \otimes \phi_{00} \otimes \phi_{j i}
$$

- Applying BNOT to the first and third Bell states results in:

$$
\sum_{i j} \frac{1}{2^{2 m}} \phi_{0 i} \otimes \phi_{00} \otimes \phi_{i j} \rightarrow \sum_{i j} \frac{1}{2^{2 m}} \phi_{00} \otimes \phi_{00} \otimes \phi_{i j}
$$


Namely, there exist a local unitary achieving the transformation

$$
\gamma^{2 m} \rightarrow \phi_{00} \otimes \phi_{00} \otimes \tau^{m}
$$

thus proving that $E_{D}\left(\gamma^{2 m}\right)=2 m$.

We now compute the bound of Corollary 19. We now have the private state:

$$
\begin{aligned}
& \mathcal{E}\left(\gamma^{2 m}\right)=\sum_{k l} \frac{1}{2^{2 m}} \phi_{0 k} \otimes \phi_{0 l} \otimes \gamma_{k l}^{2 m} \\
& \mathcal{E}\left(\hat{\gamma}^{2 m}\right)=\hat{\Phi}^{m} \otimes \hat{\Phi}^{m} \otimes \hat{\gamma}^{2 m}
\end{aligned}
$$

where:

$$
\begin{aligned}
\gamma_{k l}^{2 m} & =\sum_{i j} \frac{1}{2^{2 m}} \phi_{0, i+k} \otimes \phi_{0, j+l} \otimes \phi_{i j} \\
\hat{\gamma}_{k l}^{2 m} & =\hat{\Phi}^{m} \otimes \hat{\Phi}^{m} \otimes \tau^{m} .
\end{aligned}
$$

With the the same distillation procedure as above we can achieve the transformation

$$
\gamma_{k l}^{2 m} \rightarrow \phi_{0 k} \otimes \phi_{0 l} \otimes \tau^{m} .
$$

This allows to compute the bound of Corollary 19 as:

$$
\begin{aligned}
& \sup _{\mathcal{M}_{A}} \frac{1}{2^{2 m}} \sum_{k l} D\left(\mathcal{M}\left(\gamma_{k l}^{2 m}\right) \| \mathcal{M}\left(\hat{\gamma}^{2 m}\right)\right) \\
& =\sup _{\mathcal{M}_{A}} \frac{1}{2^{2 m}} \sum_{k l} D\left(\mathcal{M}\left(\phi_{0 k} \otimes \phi_{0 l} \otimes \tau^{m}\right) \| \mathcal{M}\left(\hat{\Phi}^{m} \otimes \hat{\Phi}^{m} \otimes \tau^{m}\right)\right) \\
& =\sup _{\mathcal{M}_{A}} \frac{1}{2^{2 m}} \sum_{k l} D\left(\mathcal{M}\left(\phi_{0 k} \otimes \phi_{0 l}\right) \| \mathcal{M}\left(\hat{\Phi}^{m} \otimes \hat{\Phi}^{m}\right)\right)=2 m
\end{aligned}
$$

achieved measuring the conjugate basis. Notice that Alice's distilling unitary can be done by the measurement, while Bob unitary can be done because of unitary invariance of the relative entropy. This bound now optimal and performs strictly better than Lemma 18.

\section{Appendix G: Key repeater}

In this Appendix we motivate the details leading to Corollary 3. The key repeater rates from [4] are defined in the same fashion as the rates from Appendix E. Recall that we now have three parties: Alice and Charlie $(A$ and $C$ ) share $\rho$ and Charlie and Bob $\left(C^{\prime}\right.$ and $\left.B\right)$ share $\rho^{\prime}$. The key repeater rate is defined as a rate at which private states can be distilled, under tripartite LOCC maps, where Charlie $\left(\boldsymbol{C}=C C^{\prime}\right)$ is traced out at the end of the protocol, see also Figure 9. Formally,

$$
R_{D}\left(\rho, \rho^{\prime}\right)=\lim _{\varepsilon \rightarrow 0} \lim _{n \rightarrow \infty} \sup _{\Lambda_{A: C: B}}\left\{R: \operatorname{tr}_{C} \Lambda\left(\left(\rho \otimes \rho^{\prime}\right)^{\otimes n}\right) \approx_{\varepsilon} \gamma^{n R}\right\},
$$

which again equals

$$
R_{D}\left(\rho, \rho^{\prime}\right)=\lim _{\varepsilon \rightarrow 0} \lim _{n \rightarrow \infty} \sup _{\Lambda_{A: C: B}}\left\{R: \operatorname{tr}_{C} \Lambda\left(\left(\rho \otimes \rho^{\prime}\right)^{\otimes n}\right) \approx_{\varepsilon} \gamma_{\text {Bell }}^{n R}\right\}
$$

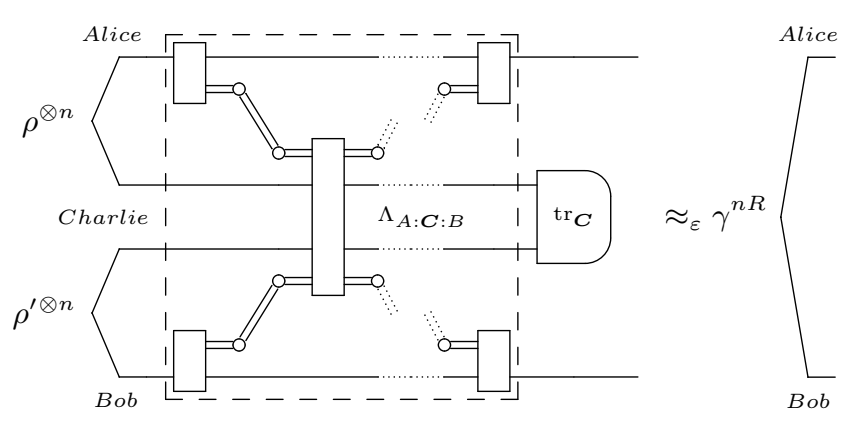

FIG. 9. Distillation of key in a single node repeater.

Let us now indicate with $L O C C_{C \rightarrow A: B}$ the tripartite LOCC protocols that have the communication between Charlie and Alice/Bob restricted to be one-way from Charlie. The corresponding one-way key repeater rate is then equal to:

$$
R_{D}\left(\rho, \rho^{\prime}\right)=\lim _{\varepsilon \rightarrow 0} \lim _{n \rightarrow \infty} \sup _{\Lambda_{C \rightarrow A: B}}\left\{R: \operatorname{tr}_{C} \Lambda\left(\left(\rho \otimes \rho^{\prime}\right)^{\otimes n}\right) \approx_{\varepsilon} \gamma^{n R}\right\} .
$$

The following reformulation is useful for the proof of the next theorem.

\section{Lemma 23.}

$$
R_{D}\left(\rho, \rho^{\prime}\right)=\lim _{\varepsilon \rightarrow 0} \lim _{n \rightarrow \infty} \sup _{\substack{\mathcal{M}_{C} \\ \Lambda_{A: B}}}\left\{R: \Lambda \circ \mathcal{M}\left(\left(\rho \otimes \rho^{\prime}\right)^{\otimes n}\right) \approx_{\varepsilon} \gamma^{n R}\right\} .
$$

Proof. An arbitrary one-way protocol $\Lambda^{\prime} \in L O C C_{C \rightarrow A: B}$ consists of an instrument on $C$ followed by an $L O C C_{A: B}$ protocol that is allowed to act on the classical part of the instrument outcome (the communication). Let $I$ be the instrument from $\boldsymbol{C}$ to $\boldsymbol{C M}$, where $M$ is now the classical register. Let $\Lambda \in L O C C_{M: A: B}$ be the second part of the protocol. Then:

$$
\begin{aligned}
\operatorname{tr}_{C} \Lambda^{\prime} & =\operatorname{tr}_{C} \circ\left(\operatorname{id}_{C} \otimes \Lambda\right) \circ\left(I \otimes \mathrm{id}_{A B}\right) \\
& =\Lambda \circ\left(\operatorname{tr}_{C} I \otimes \mathrm{id}_{A B}\right) \\
& =\Lambda \circ\left(\mathcal{M} \otimes \mathrm{id}_{A B}\right)
\end{aligned}
$$

where we used that tracing the quantum part of the instrument gives a measurement $\mathcal{M}=\operatorname{tr}_{C} I$. Since $M$ is classical we have $L O C C_{M: A: B} \equiv L O C C_{M A: B}$. Because every pair $\left(\mathcal{M}_{C}, \Lambda_{A: B}\right)$ also defines a map in $L O C C_{C \rightarrow A: B}$, we have equality in the claim.

We can now formulate the upper bound used to derive Corollary 3 , this upper bound is a corollary of the following theorem.

\section{Theorem 24 ([4, Theorem 4]).}

$$
R_{D}\left(\rho, \rho^{\prime}\right) \leq E_{R, \boldsymbol{C} \rightarrow A B}^{\infty, A: C^{\prime}: B}\left(\rho \otimes \rho^{\prime}\right) .
$$

Corollary 25. For any pair of states $\rho$ and $\rho^{\prime}$ and any separable state $\sigma$ in $S E P_{A: C B}$ or $S E P_{A C: B}$ :

$$
R_{D}\left(\rho, \rho^{\prime}\right) \leq D_{C}^{\infty}\left(\rho \otimes \rho^{\prime} \| \sigma\right) .
$$


Proof. This is a consequence of Theorem 24. The direct way of proving the claim is to first adapt the original proof to obtain:

$$
\begin{aligned}
& R_{D}^{\rightarrow}\left(\rho, \rho^{\prime}\right) \leq E_{R, \boldsymbol{C} \rightarrow A B}^{\infty, A: B}\left(\rho \otimes \rho^{\prime}\right) \\
& R_{D}^{\rightarrow}\left(\rho, \rho^{\prime}\right) \leq E_{R, \boldsymbol{C} \rightarrow A B}^{\infty, A C: B}\left(\rho \otimes \rho^{\prime}\right),
\end{aligned}
$$

then restrict the optimization over tensor product separable states $\sigma^{\otimes n}$ and finally remove the measurement on the receiver side $A B$ as done in Lemma 17.

However, we think it is instructive to see a direct proof. Without loss of generality let $\sigma$ in $\operatorname{SEP}_{A: \boldsymbol{C} B}$. According to Lemma 23, let the measurement $\mathcal{M}$ and map $\Lambda$ be the optimal $R_{D}$ distillation protocols for given $\varepsilon$ and $n$. Then, for any $\sigma \in S E P_{A: C B}$ :

$$
\begin{aligned}
\frac{1}{n} D_{\boldsymbol{C}} & \left(\left(\rho \otimes \rho^{\prime}\right)^{\otimes n} \| \sigma^{\otimes n}\right) \\
& \geq \frac{1}{n} D\left(\mathcal{M}\left(\left(\rho \otimes \rho^{\prime}\right)^{\otimes n}\right) \| \mathcal{M}\left(\sigma^{\otimes n}\right)\right) \\
& \geq \frac{1}{n} D\left(\Lambda \circ \mathcal{M}\left(\left(\rho \otimes \rho^{\prime}\right)^{\otimes n}\right) \| \Lambda \circ \mathcal{M}\left(\sigma^{\otimes n}\right)\right) \\
& \geq \frac{1}{n} D\left(\tilde{\gamma}^{n R} \| \tilde{\sigma}_{n}\right)
\end{aligned}
$$

where $\tilde{\gamma}^{n R}:=\Lambda \circ \mathcal{M}\left(\left(\rho \otimes \rho^{\prime}\right)^{\otimes n}\right)$ will be a state $\varepsilon$-close to a private state $\gamma^{n R}$, and $\tilde{\sigma}_{n}$ in still a separable state. The first inequality follows by definition of $D_{\boldsymbol{C}}$ and the second by monotonicity of the relative entropy.

Ideally, at this point we would use asymptotic continuity to change $\tilde{\gamma}^{n R}$ into $\gamma^{n R}$ at the cost of some factor that goes to zero in the limits $n \rightarrow \infty$ and $\varepsilon \rightarrow 0$. However, the dimensions of the shield systems of $\gamma^{n R}$ are in principle unbounded, so that we cannot argue directly that these factors go to zero. We need to remove the shield systems first, exploiting that, by definition, $\gamma^{n R}$ is a twisted version of a maximally entangled state $\Phi^{n R}$. This is the same argument used in [19, Theorem 9].

Let us denote by $\mathcal{T}$ the map that inverts the twisting unitary and traces the shield. Then, by monotonicity of the trace distance, we have:

$$
\mathcal{T}\left(\tilde{\gamma}^{n R}\right) \approx_{\varepsilon} \mathcal{T}\left(\gamma^{n R}\right)=\Phi^{n R}
$$

Furthermore, while $\mathcal{T}\left(\tilde{\sigma}_{n}\right)$ might not be separable anymore, $\mathcal{T}$ will still map $S E P_{A: B}$ into a convex set $\mathcal{T}(A: B)$. Again we can apply $\mathcal{T}$ by monotonicity of the relative entropy, thus we find:

$$
\begin{aligned}
\frac{1}{n} D_{\boldsymbol{C}} & \left(\left(\rho \otimes \rho^{\prime}\right)^{\otimes n} \| \sigma^{\otimes n}\right) \\
& \geq \frac{1}{n} D\left(\mathcal{T}\left(\tilde{\gamma}^{n R}\right) \| \mathcal{T}\left(\tilde{\sigma}_{n}\right)\right) \\
& \geq \frac{1}{n} E_{R}^{\mathcal{T}(A: B)}\left(\mathcal{T}\left(\tilde{\gamma}^{n R}\right)\right) \\
& \geq \frac{1}{n} E_{R}^{\mathcal{T}(A: B)}\left(\Phi^{n R}\right)-\frac{1}{n}(n R \varepsilon-\eta(\varepsilon)+\eta(1+\varepsilon)) \\
& \geq \frac{1}{n} n R-\varepsilon R+\frac{1}{n}(\eta(\varepsilon)-\eta(1+\varepsilon))
\end{aligned}
$$

$$
=(1-\varepsilon) R+\frac{1}{n}(\eta(\varepsilon)-\eta(1+\varepsilon))
$$

where we used the asymptotic continuity of the relative entropy with respect to $\mathcal{T}(A: B)$ (Lemma 11, [27, 33]), and that $E_{R}^{\mathcal{T}(A: B)}\left(\Phi^{n R}\right) \geq n R$ [19, Lemma 7]. Taking the limit $n \rightarrow \infty$ then leads to:

$$
D_{\boldsymbol{C}}^{\infty}\left(\rho \otimes \rho^{\prime} \| \sigma\right) \geq(1-\varepsilon) R
$$

and taking the limit $\varepsilon \rightarrow 0$ concludes the proof.

The original version uses states $\sigma$ separable in the quadri-partite cut $A: C: C^{\prime}: B$. However, it is immediate to see why the argument works also for $A: C B$ and $A C: B$ : the only thing required in the proof is the separability of $\Lambda(\sigma)$ in $A: B$ for any distillation protocol $\Lambda$.

\section{Appendix H: Key Swapper}

Before we begin to talk about key swapping protocols we need to introduce some definitions.

The class of Bell private states is not the only restriction one can make to the class of private states. The class of irreducible private states was defined in [19]:

Definition 26 (Irreducible private states [19]). A private state $\gamma^{m}$ is called irreducible if $K_{D}\left(\gamma^{m}\right)=m$.

These are the private states that are actually interesting in the definition of distillable key because they are the outcomes of the optimal distillation protocols. However, the only feasible way to prove that a private state is irreducible it to upper bound the distillable key via some other entanglement measure. For example one can use the relative entropy of entanglement, which in [19] it was shown to give a further upper bound in terms of the relative entropy of entanglement of the key attacked state:

$$
K_{D}\left(\gamma^{m}\right) \leq E_{R}(\gamma) \leq m+E_{R}(\hat{\gamma})
$$

In light of this technique and considering that we need to require that $\hat{\gamma}$ is separable to argue that $E_{D}=E_{R, L O C C}^{\infty}$, it is sensible to introduce the following definition:

Definition 27 (Strictly irreducible private states). We say a private state $\gamma$ is strictly irreducible if $\hat{\gamma}$ is separable. We will denote these states with $\langle\gamma\rangle$ or $\gamma^{\langle m\rangle}$.

Of course, all strictly irreducible private states are irreducible. Indeed, these are all the private states for which we can prove $K_{D}\left(\gamma^{\langle m\rangle}\right)=m$ via Equation (H1). A simple example are all private states for which $\sigma$ is the maximally mixed state $\tau$; indeed, we find immediately 
that

$$
\begin{aligned}
\hat{\gamma}^{m} & =\frac{1}{2^{m}} \sum_{i}|i i\rangle\langle i i| \otimes U_{i} \tau U_{i}^{\dagger} \\
& =\frac{1}{2^{m}} \sum_{i}|i i\rangle\langle i i| \otimes \tau U_{i} U_{i}^{\dagger} \\
& =\frac{1}{2^{m}} \sum_{i}|i i\rangle\langle i i| \otimes \tau \\
& =\hat{\Phi}^{m} \otimes \tau
\end{aligned}
$$

is always separable.

We now show that the one-way distillable entanglement upper bounds the one-way key repeater rate of all protocols that first distill strictly irreducible private states with Charlie and then try to apply a general repeater protocol. First, we define a rate for such protocols.

\section{Definition 28 (One-way key swapping rate).}

For all bipartite $\rho$ and $\rho^{\prime}$, we define the one-way key swapping rate achieved with one-way key swapping protocols as:

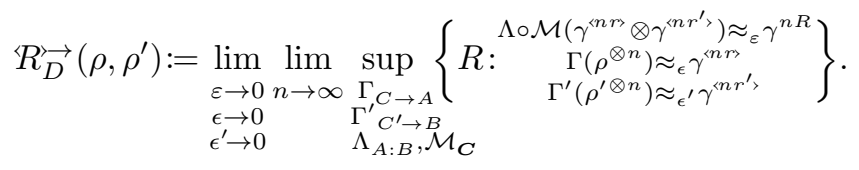

Then we can state the result:

\section{Theorem 29.}

$$
R_{D}^{\longleftrightarrow}\left(\rho, \rho^{\prime}\right) \leq E_{D}\left(\rho \otimes \rho^{\prime}\right)
$$

Proof. First notice that the tensor product of two strictly irreducible private states is still a strictly irreducible private state, namely $\gamma^{\langle a\rangle} \otimes \gamma^{\langle b\rangle}=\gamma^{\langle a+b\rangle}$.

Then, for the sake of the proof, let us introduce the following convenient bold shorthand notation:

$$
\begin{array}{rlrl}
\boldsymbol{r} & :=r+r^{\prime} & & \boldsymbol{\rho}:=\rho \otimes \rho^{\prime} \\
\boldsymbol{\gamma}^{\langle n \boldsymbol{r}\rangle}:=\gamma^{\langle n r\rangle} \otimes \gamma^{\left\langle n r^{\prime}\right\rangle} & \boldsymbol{\Gamma}:=\Gamma \otimes \Gamma^{\prime}
\end{array}
$$

then with $\boldsymbol{\epsilon}:=\epsilon+\epsilon^{\prime}$ we have

$$
\Gamma\left(\rho^{\otimes n}\right) \approx_{\epsilon} \gamma^{\langle n \boldsymbol{r}\rangle} .
$$

We also define:

$$
\mathcal{E}=\mathcal{E}_{A_{k} C_{k}} \otimes \operatorname{id}_{A_{s} C_{s}} \otimes \mathcal{E}_{B_{k} C_{k}^{\prime}} \otimes \operatorname{id}_{B_{s} C_{s}^{\prime}}
$$

where $\mathcal{E}$ is the reversible map of Lemma 1.

Proof idea: just like in the proof of Lemma 18, we exploit the idea of using the distillable entanglement as an upper bound on the protocol that performs a measurement on the shield followed by hashing. However, we need to insert a step in the proof to substitute the approximate private state with exact private state, otherwise the proofs of Corollary 19 and Theorem 2 do not work. We will do this at the level of the coherent information using its asymptotic continuity.
First, we lower bound the one-way distillable entanglement with the coherent information, just like in Corollary 19. Let $\Lambda, \mathcal{M}$ and $\boldsymbol{\Gamma}$ be the optimal key swappping maps for given $\boldsymbol{\epsilon}, \varepsilon$, and $n$ :

$$
\begin{aligned}
E_{D}(\boldsymbol{\rho}) & =\frac{1}{n} E_{D}\left(\boldsymbol{\rho}^{\otimes n}\right) \geq \frac{1}{n} E_{D}\left(\mathcal{E} \circ \boldsymbol{\Gamma}\left(\boldsymbol{\rho}^{\otimes n}\right)\right) \\
& \left.\geq \frac{1}{n} I\left(\boldsymbol{C}_{k}\right\rangle A B M\right)_{\mathcal{M} \circ \mathcal{E} \circ \boldsymbol{\Gamma}\left(\boldsymbol{\rho}^{\otimes n}\right)}
\end{aligned}
$$

where $I(X\rangle Y)=H(Y)-H(X Y)$ is the coherent information, $C_{k}=C_{k} C_{k}^{\prime}$ and $A B=A_{k} B_{k} A_{s} B_{s}$. Here we used that $E_{D}$ entanglement is a one-way LOCC monotone, that $\mathcal{E} \circ \boldsymbol{\Gamma}$ is one-way LOCC, and we used the hashing protocol after performing the measurement $\mathcal{M}$ on the shield systems at Charlie.

Here is where we want to change the approximate private states into exact private states as mentioned before. In the form of [33], asymptotic continuity of the coherent information [30] says that:

$$
\left.\mid I(X>Y)_{\varrho}-I(X\rangle Y\right)_{\varsigma}|\leq 2 \boldsymbol{\epsilon} \log | X \mid-\eta(\boldsymbol{\epsilon})+\eta(1+\boldsymbol{\epsilon})
$$

for arbitrary bipartite states such that $\varrho_{X Y} \approx_{\epsilon} \varsigma_{X Y}$; here $\eta(x)=x \log x$. Since combining Equation (H2) and the monotonicity of the trace distance gives

$$
\mathcal{M} \circ \mathcal{E}\left(\boldsymbol{\Gamma}\left(\boldsymbol{\rho}^{\otimes n}\right)\right) \approx_{\epsilon} \mathcal{M} \circ \mathcal{E}\left(\boldsymbol{\gamma}^{\langle n \boldsymbol{r}\rangle}\right),
$$

we can use the asymptotic continuity, where dimension factor is now $\log \left|\boldsymbol{C}_{k}\right|=n \boldsymbol{r}$, and get:

$$
\begin{aligned}
E_{D}(\boldsymbol{\rho}) \geq & \left.\frac{1}{n} I\left(\boldsymbol{C}_{k}\right\rangle A B M\right)_{\mathcal{M} \circ \mathcal{E}\left(\boldsymbol{\gamma}^{(n \boldsymbol{r})}\right)} \\
& -\frac{1}{n}(n \boldsymbol{r} \boldsymbol{\epsilon}-\eta(\boldsymbol{\epsilon})+\eta(1+\boldsymbol{\epsilon})) \\
= & \left.\frac{1}{n} I\left(\boldsymbol{C}_{k}\right\rangle A B M\right)_{\mathcal{M} \circ \mathcal{E}\left(\boldsymbol{\gamma}^{(n \boldsymbol{r})}\right)}+O(\boldsymbol{\epsilon}) .
\end{aligned}
$$

Now, as shown in Corollary 19 and Theorem 2, we can rewrite the conditional information as a relative entropy and then, by the unitary invariance of the relative entropy, correct the phase flip on the $A B$ side. This results in:

$$
\begin{aligned}
E_{D}(\boldsymbol{\rho}) & \geq \frac{1}{n} D\left(\mathcal{M}\left(\boldsymbol{\gamma}^{\langle n \boldsymbol{r}\rangle}\right) \| \mathcal{M}\left(\hat{\boldsymbol{\gamma}}^{\langle n \boldsymbol{r}\rangle}\right)\right)+O(\boldsymbol{\epsilon}) \\
& \geq \frac{1}{n} D\left(\Lambda \circ \mathcal{M}\left(\boldsymbol{\gamma}^{\langle n \boldsymbol{r}\rangle}\right) \| \Lambda \circ \mathcal{M}\left(\hat{\boldsymbol{\gamma}}^{\langle n \boldsymbol{r}\rangle}\right)\right)+O(\boldsymbol{\epsilon})
\end{aligned}
$$

where the last inequality holds because of the monotonicity of the relative entropy. Notice that $\Lambda \circ \mathcal{M}\left(\hat{\gamma}^{\langle n \boldsymbol{r}\rangle}\right)$ is a separable state, because we distilled to strictly irreducible private states.

At this point, just like in [19, Theorem 9] and Corollary 25 , because $\Lambda \circ \mathcal{M}\left(\boldsymbol{\gamma}^{\langle n \boldsymbol{r}\rangle}\right) \approx_{\varepsilon} \gamma^{n R}$, we can lower bound the relative entropy as follows:

$$
\begin{aligned}
E_{D}(\boldsymbol{\rho}) & \geq R-\frac{1}{n}(n R \varepsilon-\eta(\varepsilon)+\eta(1+\varepsilon))+O(\boldsymbol{\epsilon}) \\
& \geq R+O(\varepsilon)+O(\boldsymbol{\epsilon})
\end{aligned}
$$

Taking the limits $n \rightarrow \infty, \boldsymbol{\epsilon} \rightarrow 0$ and $\varepsilon \rightarrow 0$ concludes the proof. 


\section{Appendix I: Single-copy Key Repeater}

We consider now yet another variation of the repeater: the single copy key repeater rate $R_{D}^{s c}$. Instead of letting Charlie act jointly on arbitrary many copies, we restrict him to act only on a single copy of the states. This should model, for example, memory-less repeater stations that perform their operations fast and do not allow for distillation. Alice and Bob then proceed to distill key as usual with the outcome of the single copy protocol with Charlie. The single copy key repeater rate has been defined in [4] as follows.

\section{Definition 30 (Single-copy $R_{D}[4]$ ).}

$$
R_{D}^{s c}\left(\rho, \rho^{\prime}\right):=\sup _{\Lambda_{A: C: B}} K_{D}\left(\operatorname{tr}_{C} \Lambda\left(\rho \otimes \rho^{\prime}\right)\right) .
$$

It is possible to prove an upper bound in terms of a single copy relative entropy measure. We follow the proof of a similar upper bound that can be found in [4] for the general key repeater rate, the main difference is that in this case there is no regularization, which would make the bound intractable for our purposes. We will later combine this bound with Corollary 19 to express it in terms of the distillable entanglement.

Theorem 31. For all states $\rho$ and $\rho^{\prime}$ it holds:

$$
\begin{aligned}
& R_{D}^{s c}\left(\rho, \rho^{\prime}\right) \leq E_{R, \mathbb{M} \boldsymbol{C}: A B}^{A: \boldsymbol{C} B}\left(\rho \otimes \rho^{\prime}\right) \\
& R_{D}^{s c}\left(\rho, \rho^{\prime}\right) \leq E_{R, \mathbb{M} \boldsymbol{C}: A B}^{A \boldsymbol{C}:}\left(\rho \otimes \rho^{\prime}\right)
\end{aligned}
$$

where

- $E_{R, \mathbb{M} \boldsymbol{C}: A B}^{A: \boldsymbol{C}}$ and $E_{R, \mathbb{M} \boldsymbol{C}: A B}^{A: \boldsymbol{C}}$ are relative entropies with $P=S E P_{A: C B}$ and $P=S E P_{A C: B}$ respectively, and $\mathbb{L}=L O C C_{\mathbb{M C}: A B}$;

- $L O C C_{\mathbb{M} C: A B}$ are all LOCC protocols of $\boldsymbol{C}: A B$ that end with a measurement at Charlie.

Proof. Without loss of generality let $\sigma \in S E P_{A: C B}$. Furthermore, for any map $\Lambda \in L O C C_{A: C: B}$ it holds that:

$$
\begin{array}{r}
\operatorname{tr}_{C} \Lambda \in L O C C_{\mathbb{M} C: A B} \\
\tilde{\sigma}=\operatorname{tr}_{C} \Lambda(\sigma) \in S E P_{A: B} .
\end{array}
$$

Therefore, for any such $\sigma$ and $\Lambda$ we have:

$$
\begin{aligned}
D_{\mathbb{M} C: A B} & \left(\rho \otimes \rho^{\prime} \| \sigma\right) \\
& =\sup _{\mathcal{M}_{\mathbb{M C}: A B}} D\left(\mathcal{M}\left(\rho \otimes \rho^{\prime}\right) \| \mathcal{M}(\sigma)\right) \\
& \geq D\left(\operatorname{tr}_{C} \Lambda\left(\rho \otimes \rho^{\prime}\right) \| \operatorname{tr}_{C} \Lambda(\sigma)\right) \\
& =D\left(\operatorname{tr}_{C} \Lambda\left(\rho \otimes \rho^{\prime}\right) \| \tilde{\sigma}\right) \\
& \geq E_{R}\left(\operatorname{tr}_{C} \Lambda\left(\rho \otimes \rho^{\prime}\right)\right) \\
& \geq K_{D}\left(\operatorname{tr}_{C} \Lambda\left(\rho \otimes \rho^{\prime}\right)\right)
\end{aligned}
$$

where we used that $E_{R}$ is a known upper bound on $K_{D}$ [19]. Taking the supremum over all $\Lambda$ 's we find:

$$
D_{\mathbb{M} C: A B}\left(\rho \otimes \rho^{\prime} \| \sigma_{A: C B}\right) \geq R_{D}^{s c}\left(\rho, \rho^{\prime}\right) .
$$

Taking the infimum over $\sigma$ 's we end the proof.
Recall now that Corollary 19 generalizes to the two-way case in the following way:

$$
\begin{aligned}
E_{D}(\rho) & \geq \sup _{\mathcal{M}_{A} \in L O C C_{A: B}} \frac{1}{2^{m}} \sum_{j} D\left(\mathcal{M}\left(\rho_{j}\right) \| \mathcal{M}(\hat{\rho})\right) \\
& =\sup _{\mathcal{M}_{\mathbb{M} A: B}} \frac{1}{2^{m}} \sum_{j} D\left(\mathcal{M}\left(\rho_{j}\right) \| \mathcal{M}(\hat{\rho})\right)
\end{aligned}
$$

As a direct application of Corollary 20 to Theorem 31, we have now the following corollary.

Corollary 32. Let $\rho$ and $\rho^{\prime}$ be any pair of key correlated states with at least one separable key attacked state. Then:

$$
R_{D}^{s c}\left(\rho, \rho^{\prime}\right) \leq\left|B_{k}\right| \cdot E_{D}\left(\rho \otimes \rho^{\prime}\right) .
$$

Proof.

$$
\begin{aligned}
R_{D}^{s c}\left(\rho, \rho^{\prime}\right) & \leq D_{\mathbb{M} C: A B}\left(\rho \otimes \rho^{\prime} \| \hat{\rho} \otimes \hat{\rho}^{\prime}\right) \\
& =\sup _{\mathcal{M}_{\mathbb{M}: A B} D\left(\mathcal{M}\left(\rho \otimes \rho^{\prime}\right) \| \mathcal{M}\left(\hat{\rho} \otimes \hat{\rho}^{\prime}\right)\right)} \\
& \leq \sup _{\mathcal{M}_{\mathbb{M}: A B}} \sum_{j k} D\left(\mathcal{M}\left(\rho_{j} \otimes \rho_{k}^{\prime}\right) \| \mathcal{M}\left(\hat{\rho} \otimes \hat{\rho}^{\prime}\right)\right) \\
& \leq\left|B_{k}\right| \cdot E_{D}\left(\rho \otimes \rho^{\prime}\right)
\end{aligned}
$$

\section{Appendix J: Examples}

Example 33 (The Swap private states $\gamma_{\mathbb{S}}[2]$ ).

This is a class of Bell private states with $m=1$, for each dimension $d=\left|A_{s}\right|=\left|B_{s}\right|$ it defines:

$$
\gamma_{\mathbb{S}}=\frac{1}{2}\left(1+\frac{1}{d}\right) \phi_{+} \otimes \rho_{s}+\frac{1}{2}\left(1-\frac{1}{d}\right) \phi_{-} \otimes \rho_{a}
$$

for each dimension $d>1$, where $\rho_{s}$ and $\rho_{a}$ are the symmetric and anti-symmetric states in $\mathbb{C}^{d} \otimes \mathbb{C}^{d}$, the extreme Werner states [7]. In private state form, they are defined by:

$$
\sigma=\frac{\mathbb{1}}{d^{2}} \quad T=\mathbb{1}_{2} \otimes(|0\rangle\langle 0|\otimes \mathbb{1}+| 1\rangle\langle 1| \otimes \mathbb{S})
$$

where $\mathbb{S}=\sum_{i, j=0}^{d-1}|i j\rangle\langle j i|$ is the swap operator. Notice that the swap is unitary and hermitian, thus $\mathbb{S}^{\dagger} \mathbb{S}=\mathbb{S}^{\dagger}=$ $\mathbb{S}^{2}=\mathbb{1}$; this gives the following block form:

$$
\gamma_{\mathbb{S}}=\frac{1}{2} \frac{1}{d^{2}}\left[\begin{array}{llll}
\mathbb{1} & 0 & 0 & \mathbb{S} \\
0 & 0 & 0 & 0 \\
0 & 0 & 0 & 0 \\
\mathbb{S} & 0 & 0 & \mathbb{1}
\end{array}\right] .
$$

This is a strictly irreducible private state so $K_{D}\left(\gamma_{\mathbb{S}}\right)=1$. By the log-negativity upper bound on distillable entanglement [20], we have

$$
E_{D}\left(\gamma_{\mathbb{S}}\right) \leq E_{N}\left(\gamma_{\mathbb{S}}\right)=\log _{2}\left(1+\frac{1}{d}\right)
$$

which vanishes for large enough $d$.

For the Swap private states we can immediately apply Corollary 3. This is the same example from the main text. 


\section{Corollary 34.}

$$
R_{D}\left(\gamma_{\mathbb{S}}, \gamma_{\mathbb{S}}\right) \leq 2 \log _{2}\left(1+\frac{1}{d}\right) \leq 2 \frac{\log _{2} e}{d} .
$$

Aside from being the first example of an upper bound on key repeater rate for NPT states, Corollary 34 also improves on the single copy key repeater rate upper bound previously known [4]:

$$
R_{D}^{s c}\left(\gamma_{\mathbb{S}}, \gamma_{\mathbb{S}}\right) \leq O\left(\frac{\log _{2} d}{d}\right)
$$

In general, any unitary matrix in $d$ dimensions can be used to define a private state [9]. Here we focus only on the following special case:

$$
U=\sum_{i j} \frac{1}{\sqrt{d}} u_{i j}|i\rangle\langle j|
$$

such that $\left|u_{i j}\right|=1$, an example being the discrete Fourier transform. For each such $U$ we then define the following operators, to be used in the examples to follow:

$$
\begin{aligned}
\mathbb{U} & :=\sum_{i j} u_{i j}|i i\rangle\langle j j| \\
\mathbb{U}^{\Gamma} & :=\sum_{i j} u_{i j}|i j\rangle\langle j i|
\end{aligned}
$$

where $(\cdot)^{\Gamma}$ denotes the partial transpose. Notice that $\mathbb{U}^{\Gamma}$ is a unitary and $\frac{\mathbb{U}}{\sqrt{d}}$ is unitary in the maximally correlated subspace, namely

$$
\begin{aligned}
\frac{\mathbb{U}}{\sqrt{d}} \frac{\mathbb{U}^{\dagger}}{\sqrt{d}} & =\frac{\mathbb{U}^{\dagger}}{\sqrt{d}} \frac{\mathbb{U}}{\sqrt{d}}=\mathbb{1}_{\hat{\Phi}} \\
\mathbb{U}^{\Gamma^{\dagger}} \mathbb{U}^{\Gamma} & =\mathbb{U}^{\Gamma} \mathbb{U}^{\Gamma^{\dagger}}=\mathbb{1} .
\end{aligned}
$$

Example 35 (The Fourier private states $\gamma_{\mathbb{U}^{\Gamma}}[9]$ ). The class of Fourier private states defines for $m=1$, for each $d=\left|A_{s}\right|=\left|B_{s}\right|$ and for each $U$ as in Equation (J2):

$$
\sigma=\frac{\mathbb{1}}{d^{2}} \quad T=\mathbb{1}_{2} \otimes\left(|0\rangle\langle 0|\otimes \mathbb{1}+| 1\rangle\langle 1| \otimes \mathbb{U}^{\Gamma}\right)
$$

or in block form:

$$
\gamma_{\mathbb{U}^{\Gamma}}=\frac{1}{2} \frac{1}{d^{2}}\left[\begin{array}{cccc}
\mathbb{1} & 0 & 0 & \mathbb{U}^{\Gamma} \\
0 & 0 & 0 & 0 \\
0 & 0 & 0 & 0 \\
\mathbb{U}^{\Gamma^{\dagger}} & 0 & 0 & \mathbb{1}
\end{array}\right] .
$$

Notice that in general these are not Bell private states because $\mathbb{U}^{\Gamma}$ is in general not hermitian. $U$ is usually taken to be the discrete Fourier transform, thus the name.

This is also a strictly irreducible private state, thus $K_{D}\left(\gamma_{\mathbb{U}^{\Gamma}}\right)=1$, and again we have an upper bound on distillable entanglement via the log-negativity:

$$
E_{D}\left(\gamma_{\mathbb{U} \Gamma}\right) \leq E_{N}\left(\gamma_{\mathbb{U} \Gamma}\right)=\log _{2}\left(1+\frac{1}{\sqrt{d}}\right)
$$

\section{Corollary 36.}

$$
R_{D}\left(\gamma_{\mathbb{U} \Gamma}, \gamma_{\mathbb{U} \Gamma}\right) \leq 2 \log _{2}\left(1+\frac{1}{\sqrt{d}}\right) \leq 2 \frac{\log _{2} e}{\sqrt{d}}
$$

Example 37 (The Flower private states $\gamma_{\mathbb{U}}[9]$ ). Similarly, the class of Flower private states defines for $m=1$, for each $d=\left|A_{s}\right|=\left|B_{s}\right|$ and for each unitary $U$ :

$$
\sigma=\frac{\mathbb{1}_{\hat{\Phi}}}{d} \quad T=\mathbb{1}_{2} \otimes(|0\rangle\langle 0|\otimes \mathbb{1}+| 1\rangle\langle 1| \otimes \mathbb{U})
$$

or in block form:

$$
\gamma_{\mathbb{U}}=\frac{1}{2} \frac{1}{d}\left[\begin{array}{cccc}
\mathbb{1}_{\hat{\Phi}} & 0 & 0 & \frac{\mathbb{U}}{\sqrt{d}} \\
0 & 0 & 0 & 0 \\
0 & 0 & 0 & 0 \\
\frac{\mathbb{U}^{\dagger}}{\sqrt{d}} & 0 & 0 & \mathbb{1}_{\hat{\Phi}}
\end{array}\right] .
$$

Again, these are not Bell private states in general. The first such an example was the flower state [19], which is obtained when $U$ is tensor products of the Hadamard transform.

They are still strictly irreducible private states, thus $K\left(\gamma_{\mathbb{U}}\right)=1$. However, for the same reason that makes the log-negativity of the Fourier private states small, the log-negativity of the Flower private states becomes large and thus it cannot be used to find a meaningful bound on the distillable entanglement:

$$
E_{N}\left(\gamma_{\mathbb{U}}\right)=\log _{2}(1+\sqrt{d})
$$

Indeed, this can be far from the relative entropy of entanglement. Just like for the flower state, $E_{R}\left(\gamma_{\mathbb{U}}\right)=1$ because the relative entropy of entanglement is non lockable and $\gamma_{\mathbb{U}}$ becomes separable after measuring either key system in the computational basis [32]. On the other hand, we can actually compute the distillable entanglement explicitly via the hashing bound $H(B)-H(A B)$, because $\gamma_{\mathbb{U}}$ has support only on the maximally correlated subspace of $A_{k} B_{k} A_{s} B_{s}$ [31]. Since $\frac{\mathbb{U}^{\Gamma}}{\sqrt{d}}$ is a unitary in the maximally correlated subspace, it can be diagonalized in this subspace with all diagonal elements of unit module. In short, we find

$$
H\left(A_{k} B_{k} A_{s} B_{s}\right)_{\gamma_{\mathbb{U}}}=\log _{2} d
$$

while the marginals are maximally mixed so

$$
H\left(B_{k} B_{s}\right)_{\gamma_{\mathbb{U}}}=1+\log _{2} d
$$

Therefore, for the class of Flower private states:

$$
E_{D}\left(\gamma_{\mathbb{U}}\right)=R\left(\gamma_{\mathbb{U}}, \gamma_{\mathbb{U}}\right)=K\left(\gamma_{\mathbb{U}}\right)=E_{R}\left(\gamma_{\mathbb{U}}\right)=1
$$

While they might not seem interesting, we will need these states for the PPT invariant examples.

Example 38 (The PPT (noisy) private states $\left.\boldsymbol{\xi}_{\mathbb{U}} \boldsymbol{\Gamma}[4]\right)$. These are not exact private states, they are 
approximate private states that can be made arbitrarily close to the Fourier private states while still being PPT. The class of PPT private states defines (for $m=1$, for each $d=\left|A_{s}\right|=\left|B_{s}\right|$ and for each $U$ as in Equation (J2)):

$$
\xi_{\mathbb{U} \Gamma}=\frac{1}{1+\frac{1}{\sqrt{d}}}\left(\gamma_{\mathbb{U} \Gamma}+\frac{1}{\sqrt{d}} X_{A_{k}} \hat{\gamma}_{\mathbb{U}} X_{A_{k}}\right)
$$

where the function of the local bit flip is to move the key attacked state $\hat{\gamma}_{\mathbb{U}}$ in the orthogonal subspace. Namely, in block form:

$$
\xi_{\mathbb{U} \Gamma}=\frac{1}{2} \frac{1}{1+\frac{1}{\sqrt{d}}}\left[\begin{array}{cccc}
\frac{\mathbb{1}}{d^{2}} & 0 & 0 & \frac{\mathbb{U}^{\Gamma}}{d^{2}} \\
0 & \frac{1}{\sqrt{d}} \frac{\mathbb{1}_{\hat{\Phi}}}{d} & 0 & 0 \\
0 & 0 & \frac{1}{\sqrt{d}} \frac{\mathbb{1}_{\hat{\Phi}}}{d} & 0 \\
\frac{\mathbb{U}^{\Gamma \dagger}}{d^{2}} & 0 & 0 & \frac{\mathbb{1}}{d^{2}}
\end{array}\right] .
$$

One can check that this noise is just enough to make them PPT, and that remarkably, the amount of noise needed in the mixture goes to zero for large $d$. The PPT private states are engineered to become close to the set of separable states after partial transposition. Indeed, since $\mathbb{1}$ and $\mathbb{1}_{\hat{\Phi}}$ are PPT invariant, we find

$$
\xi_{\mathbb{U} \Gamma}^{\Gamma}=\frac{1}{2} \frac{1}{1+\frac{1}{\sqrt{d}}}\left[\begin{array}{ccccc}
\frac{\mathbb{1}}{d^{2}} & 0 & 0 & 0 \\
0 & \frac{1}{\sqrt{d}} \frac{\mathbb{1}_{\hat{\Phi}}}{d} & \frac{1}{\sqrt{d}} \frac{\mathbb{U}}{d \sqrt{d}} & 0 \\
0 & \frac{1}{\sqrt{d}} \frac{\mathbb{U}^{\dagger}}{d \sqrt{d}} & \frac{1}{\sqrt{d}} \frac{\mathbb{1}_{\hat{\Phi}}}{d} & 0 \\
0 & 0 & 0 & \frac{\mathbb{1}}{d^{2}}
\end{array}\right] .
$$

and thus:

$$
\xi_{\mathbb{U} \Gamma}^{\Gamma}=\frac{1}{1+\frac{1}{\sqrt{d}}}\left(\hat{\gamma}_{\mathbb{U} \Gamma}+\frac{1}{\sqrt{d}} X_{A_{k}} \gamma_{\mathbb{U}} X_{A_{k}}\right)
$$

which is suddenly mostly a separable key attacked state with a vanishing mixture of a Flower private state.

Until now, the PPT private states were the only example in the literature for which the key repeater rate could be upper bound by a computable quantity. Because these private states are PPT, the distillable entanglement is zero $\left(E_{D}\left(\xi_{\mathbb{U} \Gamma}\right)=0\right)$; however, $\xi_{\mathbb{U} \Gamma}$ are not exact private state, so we cannot use Corollary 3 directly anymore. Instead, we need to exploit the monotonicity of the key repeater rate under one-way LOCC operations and the fact that PPT private states are obtained by mixing the Fourier private states with noise via a one-way LOCC operation, thus we find:

\section{Corollary 39.}

$$
R_{D}\left(\xi_{\mathbb{U} \Gamma}, \xi_{\mathbb{U} \Gamma}\right) \leq 2 \log _{2}\left(1+\frac{1}{\sqrt{d}}\right) \leq 2 \frac{\log _{2} e}{\sqrt{d}} .
$$

Because Charlie is traced out at the end of the key repeater distillation protocol, the key repeater rate is invariant under transposition of the input on Charlie's systems, thus giving the following upper bound on the key repeater rate [4]:

$$
R_{D}\left(\rho, \rho^{\prime}\right) \leq \min \left\{K_{D}\left(\rho^{\Gamma}\right), K_{D}\left(\rho^{\prime \Gamma}\right)\right\}
$$

The previous upper bound on $R_{D}\left(\xi_{\mathbb{U}^{\Gamma}}, \xi_{\mathbb{U}^{\Gamma}}\right)$ was computed by estimating an upper bound on $K_{D}\left(\xi_{\mathbb{U}^{\Gamma}}^{\Gamma}\right)$ :

$$
R_{D}\left(\xi_{\mathbb{U} \Gamma}, \xi_{\mathbb{U} \Gamma}\right) \leq O\left(\frac{\log _{2} d}{\sqrt{d}}\right)
$$

However this bound is not optimal; used properly, Equation (J7), yields the following bound.

\section{Corollary 40.}

$$
R_{D}\left(\xi_{\mathbb{U} \Gamma}, \xi_{\mathbb{U} \Gamma}\right) \leq \frac{1}{\sqrt{d}+1}
$$

Proof. By Equation (J7) and convexity of the relative entropy of entanglement we find:

$$
\begin{aligned}
R_{D}\left(\xi_{\mathbb{U} \Gamma}, \xi_{\mathbb{U} \Gamma}\right) & \leq K_{D}\left(\xi_{\mathbb{U} \Gamma}^{\Gamma}\right) \\
& \leq E_{R}\left(\xi_{\mathbb{U} \Gamma}^{\Gamma}\right) \\
& \leq \frac{1}{1+\frac{1}{\sqrt{d}}}\left(E_{R}\left(\hat{\gamma}_{\mathbb{U} \Gamma}\right)+\frac{1}{\sqrt{d}} E_{R}\left(\gamma_{\mathbb{U}}\right)\right)
\end{aligned}
$$

However $\hat{\gamma}_{\mathbb{U} \Gamma}$ is separable and, according to Equation (J5), $E_{R}\left(\gamma_{\mathbb{U}}\right)=1$. Therefore:

$$
R_{D}\left(\xi_{\mathbb{U} \Gamma}, \xi_{\mathbb{U} \Gamma}\right) \leq \frac{1}{\sqrt{d}+1}
$$

This bound is still better than Corollary 39 and holds for two-way protocols.

Example 41 (The PPT invariant (noisy) private states $\left.\xi_{\Gamma}[9]\right)$. By substituting the key attacked state with the Flower private state in Equation (J6), the expression becomes PPT invariant. Namely, the class of PPT invariant private states defines

$$
\xi_{\Gamma}=\frac{1}{1+\frac{1}{\sqrt{d}}}\left(\gamma_{\mathbb{U} \Gamma}+\frac{1}{\sqrt{d}} X_{A_{k}} \gamma_{\mathbb{U}} X_{A_{k}}^{\dagger}\right)=\left(\xi_{\Gamma}\right)^{\Gamma}
$$

(where $X_{A_{k}}$ is the bitflip on $A_{k}$ ) with block form

$$
\xi_{\Gamma}=\frac{1}{2} \frac{1}{1+\frac{1}{\sqrt{d}}}\left[\begin{array}{cccc}
\frac{\mathbb{1}}{d^{2}} & 0 & 0 & \frac{\mathbb{U}^{\Gamma}}{d^{2}} \\
0 & \frac{1}{\sqrt{d}} \frac{\mathbb{1}_{\hat{\alpha}}}{d} & \frac{1}{\sqrt{d}} \frac{\mathbb{U}}{d \sqrt{d}} & 0 \\
0 & \frac{1}{\sqrt{d}} \frac{\mathbb{U}^{\dagger}}{d \sqrt{d}} & \frac{1}{\sqrt{d}} \frac{\mathbb{1}}{d} & 0 \\
\frac{\mathbb{U}^{\Gamma \dagger}}{d^{2}} & 0 & 0 & \frac{\mathbb{1}}{d^{2}}
\end{array}\right]
$$

which is clearly PPT invariant.

The fact that these private states are PPT invariant makes Equation (J7) useless, but a bound can still be computed combining one-way LOCC monotonicity, Corollary 3 and the Rains bound:

\section{Corollary 42.}

$$
R_{D}\left(\xi_{\Gamma}, \xi_{\Gamma}\right) \leq 2 \frac{1+\log _{2} e}{1+\sqrt{d}}
$$


Proof. We introduce the following new states:

$$
\begin{aligned}
& \alpha=\frac{1}{1+\frac{1}{\sqrt{d}}}\left(|0\rangle\left\langle 0\left|\otimes \gamma_{\mathbb{U} \Gamma}+\frac{1}{\sqrt{d}}\right| 1\right\rangle\langle 1| \otimes \gamma_{\mathbb{U}}\right) \\
& \tilde{\alpha}=\frac{1}{1+\frac{1}{\sqrt{d}}}\left(|0\rangle\left\langle 0\left|\otimes \xi_{\mathbb{U} \Gamma}+\frac{1}{\sqrt{d}}\right| 1\right\rangle\langle 1| \otimes \hat{\gamma}_{\mathbb{U}}\right) .
\end{aligned}
$$

Who holds the additional qubit is irrelevant, but by making it part of the shield it is possible to show that $\alpha$ is actually a strictly irreducible private state. Indeed the key attacked state is separable and we have

$\alpha \propto\left[\begin{array}{cccc}|0\rangle\langle 0|\otimes \mathbb{1}+| 1\rangle\langle 1| \otimes \frac{\mathbb{1}_{\hat{\Phi}}}{\sqrt{d}} & 0 & 0 & |0\rangle\left\langle 0\left|\otimes \mathbb{U}^{\Gamma}+\right| 1\right\rangle\langle 1| \otimes \mathbb{U} \\ 0 & 0 & 0 & 0 \\ 0 & 0 & 0 & 0 \\ \left(|0\rangle\left\langle 0\left|\otimes \mathbb{U}^{\Gamma}+\right| 1\right\rangle\langle 1| \otimes \mathbb{U}\right)^{\dagger} & 0 & 0 & |0\rangle\langle 0|\otimes \mathbb{1}+| 1\rangle\langle 1| \otimes \frac{\mathbb{1}_{\hat{\Phi}}}{\sqrt{d}}\end{array}\right]$

We can check that this is a private state by checking that the above matrix satisfies the block form, as explained in Appendix B. This reduces to check the following equality

$$
\begin{aligned}
& |0\rangle\langle 0|\otimes \mathbb{1}+| 1\rangle\langle 1| \otimes \frac{\mathbb{1}_{\hat{\Phi}}}{\sqrt{d}}= \\
& \quad=\sqrt{\left(|0\rangle\left\langle 0\left|\otimes \mathbb{U}^{\Gamma}+\right| 1\right\rangle\langle 1| \otimes \mathbb{U}\right)^{\dagger}\left(|0\rangle\left\langle 0\left|\otimes \mathbb{U}^{\Gamma}+\right| 1\right\rangle\langle 1| \otimes \mathbb{U}\right)}
\end{aligned}
$$

which proves that $\alpha$ is a private state. One can obtain $\xi_{\Gamma}$ from $\alpha$ via LOCC: use the additional qubit to bit flip
$\gamma_{\mathbb{U}}$ but not $\gamma_{\mathbb{U} \Gamma}$ and then trace the qubit. Furthermore, $\tilde{\alpha}$ is clearly PPT, since it is mixture of the PPT states $\xi_{\mathbb{U}^{\Gamma}}$ and $\hat{\gamma}_{\mathbb{U}}$. We find that $\alpha$ is close to $\tilde{\alpha}$ :

$$
\begin{aligned}
D(\alpha \| \tilde{\alpha}) & =\frac{1}{1+\frac{1}{\sqrt{d}}}\left(D\left(\gamma_{\mathbb{U} \Gamma} \| \xi_{\mathbb{U} \Gamma}\right)+\frac{1}{\sqrt{d}} D\left(\gamma_{\mathbb{U}} \| \hat{\gamma}_{\mathbb{U}}\right)\right) \\
& =\frac{1}{1+\frac{1}{\sqrt{d}}}\left(\log \left(1+\frac{1}{\sqrt{d}}\right)+\frac{1}{\sqrt{d}} E_{R}\left(\gamma_{\mathbb{U}}\right)\right) \\
& \leq \frac{\sqrt{d}}{1+\sqrt{d}}\left(\frac{1}{\sqrt{d} \ln 2}+\frac{1}{\sqrt{d}}\right) \\
& =\frac{1+\log _{2} e}{1+\sqrt{d}} .
\end{aligned}
$$

Now, we use one-way LOCC monotonicity of $R_{D}$, Corollary 3, $E_{D}(\rho) \leq E_{R}^{P P T}(\rho)$ [28], the fact that $\tilde{\alpha}$ is PPT and Equation (J9), in this order, to show the claim:

$$
\begin{aligned}
R_{D}\left(\xi_{\Gamma}, \xi_{\Gamma}\right) & \leq R_{D}^{\rightarrow}(\alpha, \alpha) \\
& \leq 2 E_{D}^{\rightarrow}(\alpha) \\
& \leq 2 E_{R}^{P P T}(\alpha) \\
& \leq 2 D(\alpha \| \tilde{\alpha}) \\
& \leq 2 \frac{1+\log _{2} e}{1+\sqrt{d}}
\end{aligned}
$$

